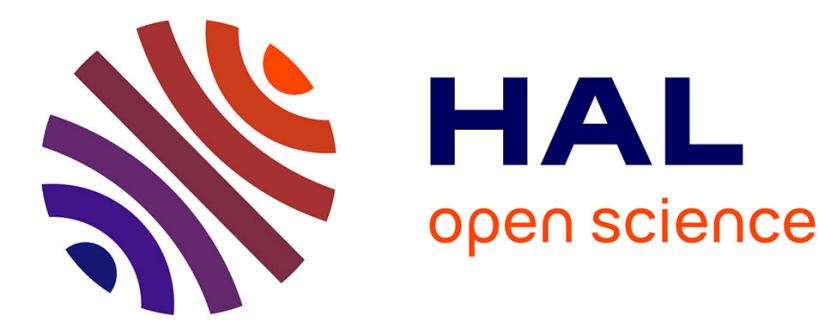

\title{
On efficiency and local uniqueness in two-sector OLG economies
}

\author{
Jean-Pierre Drugeon, Carine Nourry, Alain Venditti
}

\section{To cite this version:}

Jean-Pierre Drugeon, Carine Nourry, Alain Venditti. On efficiency and local uniqueness in two-sector OLG economies. 2009. halshs-00439241

\section{HAL Id: halshs-00439241 \\ https://shs.hal.science/halshs-00439241}

Preprint submitted on 7 Dec 2009

HAL is a multi-disciplinary open access archive for the deposit and dissemination of scientific research documents, whether they are published or not. The documents may come from teaching and research institutions in France or abroad, or from public or private research centers.
L'archive ouverte pluridisciplinaire HAL, est destinée au dépôt et à la diffusion de documents scientifiques de niveau recherche, publiés ou non, émanant des établissements d'enseignement et de recherche français ou étrangers, des laboratoires publics ou privés. 


\section{GREQAM}

Groupement de Recherche en Economie Quantitative d'Aix-Marseille - UMR-CNRS 6579 Ecole des Hautes Etudes en Sciences Sociales Universités d'Aix-Marseille II et III

\section{ON EFFICIENCY AND LOCAL UNIQUENESS}

IN TWO-SECTOR OLG ECONOMIES

\author{
Jean-Pierre DRUGEON \\ Carine NOURRY \\ Alain VENDITTI
}

September 2009
Document de Travail n'2009-28 


\title{
On efficiency and local uniqueness in two-sector OLG economies*
}

\author{
Jean-Pierre DRUGEON \\ Centre d'Economie de la Sorbonne, Centre National de la Recherche Scientifique \\ Carine NOURRY \\ Université de la Méditerranée and GREQAM \\ and \\ Alain VENDITTI \\ CNRS - GREQAM and EDHEC
}

\begin{abstract}
We consider a two-sector overlapping generations model with homothetic preferences. Under standard conditions on technologies, upon large enough values for the share of first period consumption over the wage income, we prove that dynamic efficiency and local uniqueness of the competitive equilibrium hold. On the contrary, for lower values of the share of first period consumption over the wage income which imply dynamic inefficiency of the steady state, local indeterminacy arises without requiring strong restrictions on the sectoral elasticities of capital-labor substitution.
\end{abstract}

Keywords: Two-sector OLG model, efficiency, local uniqueness.

Journal of Economic Literature Classification Numbers: C62, E32, O41.

*This work was supported by French National Research Agency Grant (ANR-08-BLAN0245-01). We thank H. d'Albis, J. Benhabib, K. Borissov, B. Decreuse, R. Farmer, C. Ghiglino, C. Hara, T. Kamihigashi, H. Kubota, N. Kudoh, T. Lloyd-Braga, T. Nakajima, O. Schmitz, T. Seegmuller for useful comments, and more specifically F. Dufourt for his many suggestions that allowed us to greatly improve the results and the exposition. This paper is based on two Working Papers by the same authors: "Does dynamic efficiency rule out expectations-driven fluctuations ?" (WP GREQAM 2006-49) and "Local indeterminacy in two-sector overlapping generations models" (WP GREQAM 2008-26). 


\section{Introduction}

Contrary to models with a finite number of infinitely-lived agents in which the competitive equilibrium is Pareto efficient and locally unique, Pareto inefficiency and local multiplicities arise whithin models with an infinite number of finitely-lived agents. ${ }^{1}$ The possibility of dynamic inefficiency in the overlapping generations (OLG) model with production has been initially demonstrated by Diamond [12]. It is related to the over-accumulation of the capital stock with respect to the Golden Rule. In other words, it is associated with too high a saving rate, and a Pareto-improvement can be achieved by allowing the current generation to devour a portion of the capital stock while leaving the consumption of all future generations intact.

In spite of the sub-optimality of the competitive equilibrium in the Diamond [12] OLG model, it is well-known that, under gross substitution in consumption, endogenous business cycle fluctuations cannot occur. In contrast, in two-sector models, endogenous fluctuations arise when the consumption good sector is capital intensive even under a gross subtitutability assumption. The input allocations across sectors, which are driven by Rybczinsky and Stolper-Samuelson effects, then generate oscillations of the capital accumulation path. $^{2}$ Moreover, local indeterminacy and expectations-driven fluctuations may also arise.

Interestingly, and as this was shown by Reichlin [22], local indeterminacy and endogenous fluctuations can occur under dynamic inefficiency in a twosector OLG model with Leontief technologies. However, a clear understanding of the relationship between dynamic efficiency and local determinacy with positive elasticities of capital-labor substitution is still missing.

The central question raised in this paper is then whether the dynamic

\footnotetext{
${ }^{1}$ See Kehoe et al. [19] and Woodford [24].

${ }^{2}$ See Galor [15] and Venditti [23].
} 
efficiency of the intertemporal allocation of capital may be a strong enough property of the competitive equilibrium to prevent the occurrence of endogenous fluctuations. The answer is positive: we prove that the conditions for dynamic efficiency rule out aggregate volatility derived from local sunspot equilibria, and that local indeterminacy arises when the steady state is dynamically inefficient. ${ }^{3}$ Our conclusions appear to be at odd with previous analysis of OLG models in which it is claimed that there is no general connection between determinacy of equilibrium and Pareto efficiency. But, all the known examples of the co-existence of local indeterminacy and Pareto efficiency are based either on pure exchange economies or on production economies in which the standard assumption of gross substitutability is violated (see for instance Kehoe and Levine [18], Woodford [24]).

We consider a formulation of the two-sector OLG model based upon standard sectoral technologies. We also assume a life-cycle utility function which is linearly homogeneous with respect to young and old consumptions so that the propensity to consume, or equivalently the share of first period consumption over the wage income, only depends on the gross rate of return on financial assets and the saving function is linear with respect to wage. Building on this property, we provide simple conditions on the propensity to consume for the existence of a steady state.

Our analysis first focuses on the dynamic efficiency properties of competitive equilibria. We show that the steady state is lower than the Golden Rule capital stock if and only if the share of first period consumption over the wage income is large enough. We also prove that under this condition, any competitive equilibrium converging to the steady state is dynamically efficient. The dynamic efficiency properties of the two-sector OLG model has not been

\footnotetext{
${ }^{3}$ Considering an aggregate OLG model augmented to include endogenous labor, Cazzavillan and Pintus [8, 9] also show that local indeterminacy is ruled out if the steady state is characterized by an under-accumulation of capital.
} 
studied until the recent contribution by Cremers [10]. She shows, as in the current paper, that it is associated with a stationary capital stock lower than the Golden Rule level. However, her results are based upon the assumption of a globally unique monotonically converging perfect foresight equilibrium, and she does not provide any conditions on the fundamentals that ensure the existence of a steady state which is exceeded by the Golden-Rule. In opposition to her contribution, our dynamic efficiency condition applies even in the case of a non-monotonic convergence process of the competitive equilibrium.

On this basis, our aim is not to provide an exhaustive analysis of the relationships between dynamic efficiency and local determinacy, but to focus on reasonable restrictions on the fundamentals. Under the standard assumptions of gross substitution in consumption and of a capital intensive consumption good, we prove in a first step that dynamic efficiency holds under three conditions: i) the share of first period consumption over the wage income is large enough, ii) the elasticity of intertemporal substitution in consumption is low enough, and iii) the sectoral elasticities of capital-labor substitution are large enough. But at the same time, we show that these conditions, which are compatible with plausible parameterizations of the fundamentals, imply the local determinacy of the generically unique steady state and thus rule out local sunspot fluctuations. In a second step, we consider the configuration with a low enough share of first period consumption over the wage income which implies that the steady state is larger than the Golden Rule capital stock and thus that the equilibrium is dynamically inefficient. We then prove that local indeterminacy easily arises without requiring strong technological restrictions but provided the elasticity of intertemporal substitution in consumption is large enough.

This paper is organized as follows: the next section sets up the basic model. In Section 3 we prove the existence of a steady state and we give conditions for dynamic efficiency of the intertemporal competitive equilibrium. 
Section 4 provides a new result on the joint occurrence of dynamic efficiency and local determinacy. Section 5 contains some concluding comments. All the proofs are given in a final Appendix.

\section{The model}

\subsection{Consumption and savings}

The economy is populated by finitely-lived agents. In each period $t, N_{t}$ persons are born, and they live for two periods. In their first period of life (when young), the agents are endowed with one unit of labor that they supply inelastically to firms. Their income directly results from the real wage. They allocate this income between current consumption and savings which are invested in the firms. In their second period of life (when old), they are retired. Their income is given by the return on the savings made at time $t$. As they do not care about events occurring after their death, they consume their income entirely. The preferences of a representative agent born at time $t$ are thus defined over his consumption bundle $\left(c_{t}\right.$, when he is young, and $d_{t+1}$, when he is old) and are summarized by the utility function $u\left(c_{t}, d_{t+1} / B\right)$, with $B>0$ a scaling constant.

Assumption 1. $u(c, d / B)$ is $\mathbf{C}^{2}$ over $\mathbb{R}_{+}^{2}$, increasing with respect to each argument $\left(u_{1}(c, d / B)>0, u_{2}(c, d / B)>0\right)$, concave over $\mathbb{R}_{++}^{2}$ and homogeneous of degree one. Moreover, for all $c, d>0, \lim _{d / c B \rightarrow 0} u_{1} / u_{2}=0$ and $\lim _{d / c B \rightarrow+\infty} u_{1} / u_{2}=+\infty$, where $u_{1} / u_{2}$ stands for $u_{1}(1, d / c B) / u_{2}(1, d / c B){ }^{4}$

Each agent is assumed to have $1+n>0$ children so that population is increasing at constant rate $n$, i.e., $N_{t+1}=(1+n) N_{t}$. Under perfect foresight,

\footnotetext{
${ }^{4}$ Note that with homothetic preferences such that $U(c, d / B)=f(u(c, d / B))$, with $f(x)$ monotone increasing and $u(c, d / B)$ linear homogeneous, all our results still holds.
} 
and considering $w_{t}$ and $R_{t+1}$ as given, a young agent maximizes his utility function over his life-cycle as follows:

$$
\begin{array}{cl}
\max _{c_{t}, d_{t+1}, \phi_{t}} & u\left(c_{t}, d_{t+1} / B\right) \\
\text { s.t. } & w_{t}=c_{t}+\phi_{t}, R_{t+1} \phi_{t}=d_{t+1}, \text { and } c_{t}, d_{t+1}, \phi_{t} \geq 0
\end{array}
$$

Assumption 1 implies the existence and uniqueness of interior solutions for optimal saving $\phi_{t}$. Using the homogeneity of $u\left(c_{t}, d_{t+1} / B\right)$, the first order conditions state as:

$$
\begin{aligned}
\frac{u_{1}\left(1, d_{t+1} / c_{t} B\right)}{u_{2}\left(1, d_{t+1} / c_{t} B\right)} & \equiv g\left(d_{t+1} / c_{t} B\right)=R_{t+1} / B \\
c_{t}+\frac{d_{t+1}}{R_{t+1}} & =w_{t} \\
\phi_{t} & =w_{t}-c_{t}
\end{aligned}
$$

The normality of $c_{t}$ implies $g^{\prime}(d / c B)>0$ and we get under Assumption 1:

$$
c_{t}=\frac{u_{1}\left(1, g^{-1}\left(R_{t+1} / B\right)\right)}{u\left(1, g^{-1}\left(R_{t+1} / B\right)\right)} w_{t} \equiv \alpha\left(R_{t+1} / B\right) w_{t}
$$

with $\alpha(R / B) \in(0,1)$ the propensity to consume of the young, or equivalently the share of first period consumption over the wage income. We also conclude that the first order condition (4) becomes:

$$
\phi_{t}=\phi\left(w_{t}, R_{t+1} / B\right) \equiv\left(1-\alpha\left(R_{t+1} / B\right)\right) w_{t}
$$

In the rest of the paper we introduce the following standard Assumption:

Assumption 2. The consumption levels $c_{t}$ and $d_{t+1}$ are gross substitutes.

Such a restriction implies that the saving function (6) is increasing with respect to the gross rate of return $R$. Note also that Assumption 2 is satisfied if and only if the elasticity of intertemporal substitution in consumption $\gamma(R / B)$, as given by: ${ }^{5}$

is larger than 1 .

$$
\gamma(R / B)=\frac{u_{1}\left(1, g^{-1}(R / B)\right) u_{2}\left(1, g^{-1}(R / B)\right)}{u\left(1, g^{-1}(R / B)\right) u_{12}\left(1, g^{-1}(R / B)\right)},
$$

\footnotetext{
${ }^{5}$ Taking the elasticity of $(2)$, and considering the Euler identity applied to $u_{1}$, i.e., $u_{11}\left(1, g^{-1}(R / B)\right)+u_{12}\left(1, g^{-1}(R / B)\right) g^{-1}(R / B) \equiv 0$, generate $\gamma(R / B)$ as the elasticity of $g^{-1}(R / B)$.
} 


\subsection{Production}

There are two produced goods, one consumption good $y_{0}$ and one capital good $y$. The consumption good, which cannot be used as capital, is entirely consumed, and the capital good cannot be consumed. There are two inputs, capital and labor. We assume complete depreciation of capital within one period and also that labor is inelastically supplied. Each good is produced with a standard constant returns to scale technology:

$$
y_{0}=f^{0}\left(k^{0}, l^{0}\right), \quad y=f^{1}\left(k^{1}, l^{1}\right)
$$

with $k^{0}+k^{1} \leq k, k$ being the total stock of capital, and $l^{0}+l^{1} \leq \ell, \ell$ being the total amount of labor.

Assumption 3. Each production function $f^{i}: \mathbb{R}_{+}^{2} \rightarrow \mathbb{R}_{+}, i=0,1$, is $C^{2}$, increasing in each argument, concave, homogeneous of degree one and such that for any $x>0, f_{1}^{i}(0, x)=f_{2}^{i}(x, 0)=+\infty, f_{1}^{i}(+\infty, x)=f_{2}^{i}(x,+\infty)=0$.

By definition we have $y \leq f^{1}(k, \ell)$. The monotonicity properties and the Inada conditions in Assumption 3 then imply that there is $\bar{k}(\ell)>0$ solution of $k-f^{1}(k, \ell)=0$ such that $f^{1}(k, \ell)>k$ when $k<\bar{k}(\ell)$, and $f^{1}(k, \ell)<k$ when $k>\bar{k}(\ell)$. It follows that it is not possible to maintain stocks beyond $\bar{k}(\ell)$. The set of admissible 3 -uples $(k, y, \ell)$ is thus defined as follows

$$
\tilde{\mathcal{K}}=\left\{(k, y, \ell) \in \mathbb{R}_{+}^{3} \mid 0<\ell, 0 \leq k \leq \bar{k}(\ell), 0 \leq y \leq f^{1}(k, \ell)\right\}
$$

There are two representative firms, one for each sector. For any given $(k, y, \ell)$, profit maximization is equivalent to solving the following problem of optimal allocation of productive factors between the two sectors:

$$
\begin{aligned}
T(k, y, \ell)=\max _{k^{0}, k^{1}, l^{0}, l^{1}} & f^{0}\left(k^{0}, l^{0}\right) \\
\text { s.t. } & y \leq f^{1}\left(k^{1}, l^{1}\right) \\
& k^{0}+k^{1} \leq k \\
& l^{0}+l^{1} \leq \ell \\
& k^{0}, k^{1}, l^{0}, l^{1} \geq 0
\end{aligned}
$$


The social production function $T(k, y, \ell)$ describes the frontier of the production possibility set associated with interior temporary equilibria $(k, y, \ell) \in \tilde{\mathcal{K}}$, and gives the maximal output of the consumption good. Under Assumption 3 , for any $(k, y, \ell) \in \tilde{\mathcal{K}}, T(k, y, \ell)$ can be shown to be homogeneous of degree one, concave and twice continuously differentiable. ${ }^{6}$ Denoting $w$ the wage rate, $r$ the gross rental rate of capital and $p$ the price of investment good, all in terms of the price of the consumption good, we may formulate the aggregate profit maximization problem as follows

$$
\max _{(k, y, \ell) \in \tilde{\mathcal{K}}} T(k, y, \ell)+p y-r k-w \ell
$$

and we derive that for any $(k, y, \ell) \in \operatorname{int}(\tilde{\mathcal{K}})$, the first-order derivatives of the social production function give

$$
r=T_{1}(k, y, \ell), \quad p=-T_{2}(k, y, \ell), w=T_{3}(k, y, \ell)
$$

\subsection{Perfect-foresight competitive equilibrium}

Total labor is given by the number $N_{t}$ of young households, i.e., $\ell_{t}=N_{t}$, and is thus increasing at rate $n$, i.e., $\ell_{t+1}=(1+n) \ell_{t}$. We then define a perfect-foresight competitive equilibrium:

Definition 1. A sequence $\left\{k_{t}, y_{t}, \ell_{t}, c_{t}, d_{t}, r_{t}, w_{t}, p_{t}\right\}_{t=0}^{\infty}$, with $\left(k_{0}, \ell_{0}\right)=$ $\left(\hat{k}_{0}, \hat{\ell}_{0}\right)$ given, is a perfect-foresight competitive equilibrium if:

i) $\left(k_{t}, y_{t}, \ell_{t}\right)$ solves (10) for any $t \geq 0$ and $\left(r_{t}, w_{t}, p_{t}\right)$ is given by (11) with $R_{t+1}=r_{t+1} / p_{t}{ }^{7}$

ii) $c_{t}=\alpha\left(R_{t+1} / B\right) w_{t}$,

iii) $\ell_{t}\left(1-\alpha\left(R_{t+1} / B\right)\right) w_{t}=p_{t} y_{t}$

\footnotetext{
${ }^{6}$ See Benhabib and Nishimura [2].

${ }^{7}$ Starting from the equality $\ell_{t}\left[c_{t}+d_{t} /(1+n)\right]=T\left(k_{t}, y_{t}, \ell_{t}\right)$ and using the budget constraints in (1) with the homogeneity of $T(k, y, \ell)$ we get $\ell_{t+1}\left(w_{t+1}-\phi_{t+1}+R_{t+1} \phi_{t}\right)=$ $r_{t+1} k_{t+1}-p_{t+1} y_{t+1}+w_{t+1} \ell_{t+1}$. The result is obtained after obvious simplifications.
} 
iv) $y_{t}=k_{t+1}$,

v) $\ell_{t+1}=(1+n) \ell_{t}$

vi) $\ell_{t}\left[c_{t}+d_{t} /(1+n)\right]=T\left(k_{t}, y_{t}, \ell_{t}\right)$.

Let us denote $\kappa_{t}=k_{t} / \ell_{t}$ the capital-labor ratio at time $t \geq 0$ and let $\bar{\kappa} \equiv$ $\bar{k}(\ell) / \ell$ be the maximal admissible value of $\kappa$. We can then redefine the set of admissible paths given by (8) as follows

$$
\mathcal{K}=\left\{\left(\kappa_{t}, \kappa_{t+1}\right) \in \mathbb{R}_{+}^{2} \mid 0 \leq \kappa_{t} \leq \bar{\kappa}, 0 \leq \kappa_{t+1} \leq f^{1}\left(\kappa_{t}, 1\right) /(1+n)\right\}
$$

Using the linear homogeneity of $T(k, y, \ell)$, we derive from Definition 1 that a perfect-foresight competitive equilibrium satisfies the following equation:

$$
(1+n) \kappa_{t+1}+\frac{T_{3}\left(\kappa_{t},(1+n) \kappa_{t+1}, 1\right)}{T_{2}\left(\kappa_{t},(1+n) \kappa_{t+1}, 1\right)}\left[1-\alpha\left(-\frac{T_{1}\left(\kappa_{t+1},(1+n) \kappa_{t+2}, 1\right)}{T_{2}\left(\kappa_{t},(1+n) \kappa_{t+1}, 1\right) B}\right)\right]=0
$$

with $\left(\kappa_{t}, \kappa_{t+1}\right) \in \operatorname{int}(\mathcal{K})$ and $\kappa_{0}=\hat{\kappa}_{0}=\hat{k}_{0} / \hat{\ell}_{0}$ given.

\section{$3 \quad$ Steady state and dynamic efficiency}

\subsection{Existence}

A steady state is defined as $\kappa_{t}=\kappa^{*}, p_{t}=p^{*}=-T_{2}\left(\kappa^{*},(1+n) \kappa^{*}, 1\right), r_{t}=$ $r^{*}=T_{1}\left(\kappa^{*},(1+n) \kappa^{*}, 1\right), w_{t}=w^{*}=T_{3}\left(\kappa^{*},(1+n) \kappa^{*}, 1\right)$ and $R^{*}=r^{*} / p^{*}$ for all $t$ with $\kappa^{*}$ solution of the following equation

$$
(1+n) \kappa^{*}+\frac{T_{3}\left(\kappa^{*},(1+n) \kappa^{*}, 1\right)}{T_{2}\left(\kappa^{*},(1+n) \kappa^{*}, 1\right)}\left[1-\alpha\left(-\frac{T_{1}\left(\kappa^{*},(1+n) \kappa^{*}, 1\right)}{T_{2}\left(\kappa^{*},(1+n) \kappa^{*}, 1\right) B}\right)\right]=0
$$

We will consider in the following a family of economies parameterized by the elasticity of intertemporal substitution in consumption $\gamma(R / B)$. The steady state $\kappa^{*}$ clearly depends on the characteristics of technologies and preferences. As a result, varying $\gamma(R / B)$ generates modifications of the value of the stationary capital-labor ratio and thus implies variations of all the other shares and elasticities characterizing the technologies and preferences. This property significantly complicates the local stability and bifurcation analysis. To simplify, we follow the same procedure as in Lloyd-Braga et 
al. [20]: building on the homogeneity property of the utility function, we use the scaling parameter $B$ in order to give conditions for the existence of a normalized steady state $\kappa^{*} \in(0, \bar{\kappa})$ which will remain invariant as the elasticity of intertemporal substitution in consumption is varied. Therefore, for a given set of parameters characterizing the technologies and preferences, we will be able to isolate the role of $\gamma(R / B)$ on the local stability properties of competitive equilibria.

Let us denote $z=R / B$. Under Assumption 2, the share of first period consumption $\alpha(z)$ is a monotone decreasing function with $\lim _{z \rightarrow 0} \alpha(z)=\alpha_{\text {sup }}$ and $\lim _{z \rightarrow+\infty} \alpha(z)=\alpha_{\text {inf }}$. By definition, we have $\left(\alpha_{\text {inf }}, \alpha_{\text {sup }}\right) \subseteq(0,1)$. Now let us define from $(13):^{8}$

$$
\Phi_{\kappa^{*}}=1+(1+n) \frac{\kappa^{*} T_{2}\left(\kappa^{*},(1+n) \kappa^{*}, 1\right)}{T_{3}\left(\kappa^{*},(1+n) \kappa^{*}, 1\right)} \in(0,1)
$$

By choosing appropriately the value of $\kappa^{*} \in(0, \bar{\kappa})$, we may derive a corresponding $\Phi_{\kappa^{*}} \in\left(\alpha_{\text {inf }}, \alpha_{\text {sup }}\right)$. We then get:

Proposition 1. Under Assumptions 1-3, let $\kappa^{*} \in(0, \bar{\kappa})$ be such that $\Phi_{\kappa^{*}} \in$ $\left(\alpha_{\text {inf }}, \alpha_{\text {sup }}\right)$. Then there is a unique value $B\left(\kappa^{*}\right)>0$ for the scaling parameter solution of

$$
\alpha\left(-\frac{T_{1}\left(\kappa^{*},(1+n) \kappa^{*}, 1\right)}{T_{2}\left(\kappa^{*},(1+n) \kappa^{*}, 1\right) B}\right)=\Phi_{\kappa^{*}}
$$

such that $\kappa^{*}$ is a steady state if and only if $B=B\left(\kappa^{*}\right)$.

Proof: See Appendix 6.1.

In the rest of the paper we will assume that the scaling parameter $B$ adjusts to $B=B\left(\kappa^{*}\right)$ in order to guarantee the existence of one normalized steady state (NSS in the sequel). Indeed, as $\gamma(R / B)$ is made to vary, the NSS and thus the share of first period consumption $\alpha(R / B)$ remain constant.

${ }^{8}$ From Definition 1 , we indeed derive $1+(1+n) \kappa^{*} T_{2}\left(\kappa^{*},(1+n) \kappa^{*}, 1\right) / T_{3}\left(\kappa^{*},(1+\right.$ $\left.n) \kappa^{*}, 1\right)=1-p y / w l=1-\phi / w \in(0,1)$. 


\subsection{Dynamic efficiency}

Our aim is to analyze the dynamic efficiency properties of the competitive equilibrium around the NSS. From (5), (7), (16), and $B=B\left(\kappa^{*}\right)$, let $\alpha=$ $\alpha\left(-T_{1}\left(\kappa^{*},(1+n) \kappa^{*}, 1\right) / T_{2}\left(\kappa^{*},(1+n) \kappa^{*}, 1\right) B\left(\kappa^{*}\right)\right)$ and $\gamma=\gamma\left(-T_{1}\left(\kappa^{*},(1+\right.\right.$ $\left.\left.n) \kappa^{*}, 1\right) / T_{2}\left(\kappa^{*},(1+n) \kappa^{*}, 1\right) B\left(\kappa^{*}\right)\right)$. Using definition 1 and the homogeneity of $T(k, y, \ell)$, we get $\kappa^{*} T_{2} / T_{3}=\left(T_{2} / T_{1}\right)\left(\kappa^{*} T_{1} / T_{3}\right)=-s / R^{*}(1-s)$, with $s=s\left(\kappa^{*},(1+n) \kappa^{*}, 1\right)$ the share of capital in total income as given by

$$
s\left(\kappa^{*},(1+n) \kappa^{*}, 1\right)=\frac{r \kappa^{*}}{T\left(\kappa^{*},(1+n) \kappa^{*}, 1\right)+p(1+n) \kappa^{*}} \in(0,1),
$$

and we derive from (15) the stationary gross rate of return along the NSS:

$$
R^{*}=\frac{(1+n) s}{(1-\alpha)(1-s)}
$$

It is well-known since Diamond [12] that if too much capital is accumulated in the long run, the economy is dynamically inefficient. Such a situation occurs if the population growth factor $1+n$ exceeds the steady state marginal product of capital. Following Phelps [21], it is then said that the capital-labor ratio exceeds the Golden Rule level. In a two-sector OLG model, the Golden Rule level of capital-labor ratio, denoted $\hat{\kappa}$, is characterized from the total stationary consumption which is given by the social production function:

$$
c+d /(1+n)=T(\kappa,(1+n) \kappa, 1)
$$

Along a stationary path of capital, the highest utility is defined as the maximum of $u(c, d)$ subject to (18). There is no other restriction than the nonnegativity of capital and consumptions, and the maximum of utility implies the maximum of the consumption good's output $T(\kappa,(1+n) \kappa, 1)$. As in the aggregate Diamond formulation [12], the Golden-Rule capital-labor ratio $\hat{\kappa}$ is independent of the intertemporal allocation of consumption:

Proposition 2. Under Assumptions 1-3, there is a unique optimal stationary path $(\hat{\kappa}, \hat{c}, \hat{d})$ which is characterized by the following conditions:

$$
R(\hat{\kappa}) \equiv \hat{R}=1+n, \hat{c}+\frac{\hat{d}}{1+n}=T(\hat{\kappa},(1+n) \hat{\kappa}, 1), u_{1}(\hat{c}, \hat{d})=(1+n) u_{2}(\hat{c}, \hat{d})
$$

with $\hat{\kappa}$ the Golden-Rule capital-labor ratio. 
Proof: See Appendix 6.2.

We now characterize the dynamic efficiency properties of equilibrium paths. Building on Proposition 2, they are appraised through the comparison of the NSS with respect to the Golden Rule. Indeed, the concept of efficiency is introduced following Cass [7]. Obviously, any efficient path needs to be feasible. Hence, a path of capital stocks per capita $\left\{\kappa_{t}\right\}_{t \geq 0}$ is called feasible if, for all $t \geq 0,\left(\kappa_{t}, \kappa_{t+1}\right) \in \mathcal{K}$ and $T\left(\kappa_{t},(1+n) \kappa_{t+1}, 1\right) \geq 0$. A feasible path of capital stocks per capita $\left\{\kappa_{t}\right\}_{t \geq 0}$ is inefficient if there is another feasible path $\left\{\kappa_{t}^{\prime}\right\}_{t \geq 0}$ such that, on the one hand, $\kappa_{0}^{\prime}=\kappa_{0}$ and for any $t \geq 0$, $T\left(\kappa_{t}^{\prime},(1+n) \kappa_{t+1}^{\prime}, 1\right) \geq T\left(\kappa_{t},(1+n) \kappa_{t+1}, 1\right)$, and, on the other hand, there is $t \geq 0$ such that $T\left(\kappa_{t}^{\prime},(1+n) \kappa_{t+1}^{\prime}, 1\right)>T\left(\kappa_{t},(1+n) \kappa_{t+1}, 1\right)$. As a result, a feasible path is efficient if it is not inefficient.

Considering the stationary gross rate of return as defined by (17), we then derive a condition on the share of first period consumption over the wage income $\alpha$ to get a NSS lower than the Golden Rule level and to ensure the dynamic efficiency of equilibria:

Proposition 3. Under Assumptions 1-3, let $\hat{\alpha}=1-s /(1-s)$. Then:

i) the NSS is characterized by an under-accumulation of capital if and only if the share of first period consumption over the wage income is sufficiently large, namely $\alpha \geq \hat{\alpha}$, i.e., if and only if $R^{*} \geq \hat{R}=1+n$;

ii) an intertemporal competitive equilibrium converging towards the NSS is dynamically efficient if $\alpha \in(\hat{\alpha}, 1)$ and dynamically inefficient if $\alpha \in(0, \hat{\alpha}){ }^{9}$

Proof: See Appendix 6.3.

Proposition 3 shows that if the labor income is relatively lower than the capital income, i.e., $s \geq 1 / 2$, then a young agent does not have enough wage

\footnotetext{
${ }^{9}$ Dynamic efficiency in the exceptional case when the stationary capital-labor ratio is just equal to the Golden Rule is a difficult problem that has been analyzed by Cass [7].
} 
resources to provide a large amount of savings so that an under-accumulation of capital is obtained without additional restriction. On the other hand, if the labor income is relatively larger than the capital income, i.e., $s<1 / 2$, then a young agent receives enough wage resources to be able to provide a large amount of savings. In this case, over-accumulation of capital can be avoided provided his share of first period consumption over the wage income is large enough. Note also that our condition for an under-accumulation of capital $\alpha \geq \hat{\alpha}$ can be equivalently reformulated as in Phelps [21], namely the aggregate saving rate $\varsigma \equiv(1-\alpha) w /(w+r k)=(1-\alpha)(1-s)$ needs to be lower than the share of capital in total income $s$, i.e., $\varsigma \leq s$.

The criterion for dynamic efficiency provided in Proposition 3 is first based upon a NSS which is characterized by an under-accumulation of capital, i.e., a large enough amount of first period consumption. Second, it is based upon a NSS which is stable. Our model consists in one predetermined variable, the current capital stock, and one forward variable, the next period capital stock. Therefore, stability of the NSS can be understood in two different ways: if the dimension of the stable manifold is equal to one, then the NSS is saddle-point stable. For a given initial capital stock, there is a unique converging equilibrium path. In such a case, the NSS is locally determinate. ${ }^{10}$ If, on the contrary, the dimension of the stable manifold is equal to two, there exists a continuum of equilibrium paths starting from the same initial capital stock and converging to the NSS. In this case, the NSS is locally indeterminate. The dynamic efficiency property of the NSS will be appraised through these two types of stability.

\footnotetext{
${ }^{10}$ If the dimension of the stable manifold is equal to zero, the NSS is totally unstable but we still call it locally determinate as there exists no equilibrium path converging to it.
} 


\section{Dynamic efficiency and local determinacy}

\subsection{The approach}

Let us introduce the relative capital intensity difference across sectors

$$
b \equiv \frac{l^{1}}{y}\left(\frac{k^{1}}{l^{1}}-\frac{k^{0}}{l^{0}}\right)
$$

and the elasticity of the rental rate of capital

$$
\varepsilon_{r k}=-T_{11}\left(\kappa^{*},(1+n) \kappa^{*}, 1\right) \kappa^{*} / T_{1}\left(\kappa^{*},(1+n) \kappa^{*}, 1\right)
$$

evaluated at the NSS. Note that the elasticity $\varepsilon_{r k}$ is introduced for analytical tractability. However, as shown in Drugeon [13], we can relate $\varepsilon_{r k}$ to the sectoral elasticities $\sigma_{0}$ and $\sigma_{1}$ of capital-labor substitution, namely:

$$
\varepsilon_{r k}=\left(l_{0} / y_{0}\right)^{2} \frac{w p y k y_{0}}{p y k_{0} l_{0} \sigma_{0}+y_{0} k_{1} l_{1} \sigma_{1}}
$$

It follows that $\varepsilon_{r k}$ is a decreasing function of $\sigma_{0}$ and $\sigma_{1}$.

Proposition 1 shows that when the scaling parameter satisfies $B=B\left(\kappa^{*}\right)$, the NSS $\kappa^{*}$ and the share of first period consumption over the wage $\alpha=\Phi_{\kappa^{*}}$ remain constant as the elasticity of intertemporal substitution $\gamma$ is made to vary. As in Grandmont et al. [16], and under the gross substitutability Assumption 2, we will then study the variations of the trace $\mathcal{T}(\gamma)$ and the determinant $\mathcal{D}(\gamma)$ in the $(\mathcal{T}, \mathcal{D})$ plane as $\gamma$ varies continuously within $(1,+\infty)$. Assuming that $b \neq 0$, linearizing the difference equation (13) around the NSS and solving $\mathcal{T}$ and $\mathcal{D}$ (as given in Appendix 6.4) with respect to $\alpha(\gamma-1)$ yields the following linear relationship $\Delta(\mathcal{T})$ :

$$
\mathcal{D}=\Delta(\mathcal{T})=\mathcal{S} \mathcal{T}-\frac{\varepsilon_{r k} s[1-\alpha+\alpha(1+n) b]-s(1+n) b}{(1+n) b\left[(1-\alpha)(1-s)+\varepsilon_{r k} s(1+n) b(1-\alpha+\alpha(1+n) b)\right]}
$$

where the slope $\mathcal{S}$ of $\Delta(\mathcal{T})$ is

$$
\mathcal{S}=\frac{\varepsilon_{r k} s[1-\alpha+\alpha(1+n) b]}{(1-\alpha)(1-s)+\varepsilon_{r k} s(1+n) b[1-\alpha+\alpha(1+n) b]} .
$$

As $\gamma$ spans the interval $(1,+\infty), \mathcal{T}(\gamma)$ and $\mathcal{D}(\gamma)$ vary linearly along the line $\Delta(\mathcal{T})$. When different values for $s, \alpha, b$ and $\varepsilon_{r k}$ are considered, the location of $\Delta(\mathcal{T})$ on the $(\mathcal{T}, \mathcal{D})$ plane is modified so that all the possible configurations for the stability properties of the NSS can be derived. 


\subsection{The determinacy properties of the steady state}

Under gross substitutability, i.e., $\gamma>1$, the NSS is locally determinate as soon as $b \geq 0$ since in this case $\mathcal{D}>1$ (see Appendix 6.4). Therefore, local indeterminacy of the NSS, which is necessarily based on $\mathcal{D}<1$, requires a capital intensive consumption good, i.e., $b<0$. In the rest of the paper we will then focus on this configuration and we will also restrict the share of capital in total income in order to get a positive value for the bound $\hat{\alpha}=1-s /(1-s)$ :

Assumption 4. $b<0$ and $s \in(0,1 / 2)$.

\subsubsection{Dynamic efficiency and local uniqueness}

Let us start with a simple configuration in which we assume a slightly stronger condition than the one ensuring an under-accumulation of capital.

Proposition 4. Under Assumptions 1-4, let the NSS be characterized by an under-accumulation of capital with $\alpha>\max \{\hat{\alpha}, 1 / 2\}$. Then the NSS is locally determinate.

Proof: See Appendix 6.5.

In two-sector models with a gross substitutability assumption, the existence of endogenous fluctuations is fundamentally based upon a capitalintensive consumption good sector. The input allocations across sectors, which are driven by Rybczinsky and Stolper-Samuelson effects, indeed generate oscillations of the capital accumulation path which may be large enough to be propagated in the economy through the savings behavior of the agents. However, when $\alpha>\max \{\hat{\alpha}, 1 / 2\}$, the propensity to save is too low so that the amount of capital accumulated is not sufficient to lead to amplified fluctuations based upon self-fulfilling expectations. Such a mechanism is also based upon a trade-off between the value of the share of capital in total 
income $s$ and the value of the share of first period consumption in wage income $\alpha$. Indeed, when $s \leq 1 / 3$, we get $\hat{\alpha} \geq 1 / 2$ and the condition of Proposition 4 simply becomes $\alpha>\hat{\alpha}$. On the contrary, when $s \in(1 / 3,1 / 2)$, local indeterminacy is ruled out if $\alpha>1 / 2(>\hat{\alpha})$.

Proposition 4 provides a link between under-accumulation of capital and local determinacy. However, Proposition 3 shows that dynamic efficiency is related to the stability of the equilibrium path. As local determinacy is compatible with a saddle-point stable or a totally unstable NSS, we need to find conditions for the saddle-point stability of the NSS.

As shown in Appendix 6.5, three critical bounds on $b$ are crucial for the stability properties of the NSS:

$$
b_{0}=-\frac{1}{1+n}, \quad b_{1}=\frac{1-\alpha}{\alpha} b_{0}, \quad b_{2}=\frac{(1-\alpha)(1-s)}{s} b_{0}
$$

Note that when $\alpha>\max \{\hat{\alpha}, 1 / 2\}, b_{0}<b_{1}, b_{2}$. There also exists a critical bound $\bar{\varepsilon}_{r k}>0$ for the elasticity of the rental rate of capital which has to be considered in some cases. In Figure 1 we first assume extreme values for $b$ with $\bar{b}=\max \left\{b_{1}, b_{2}\right\}$ and $b \in\left(-\infty, b_{0}\right) \cup(\bar{b}, 0)$.

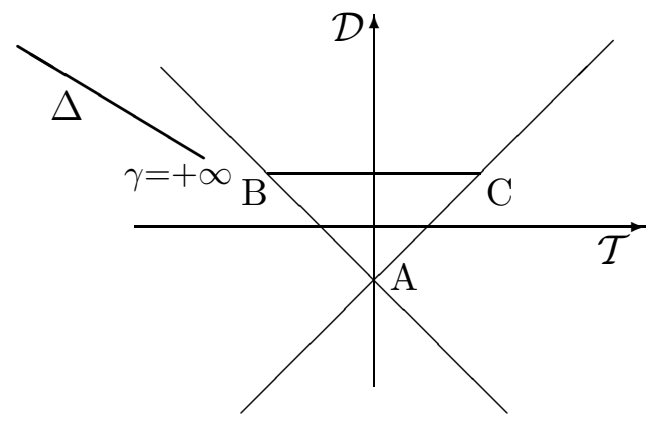

$1-$ i) $b<-\frac{1}{1+n}$

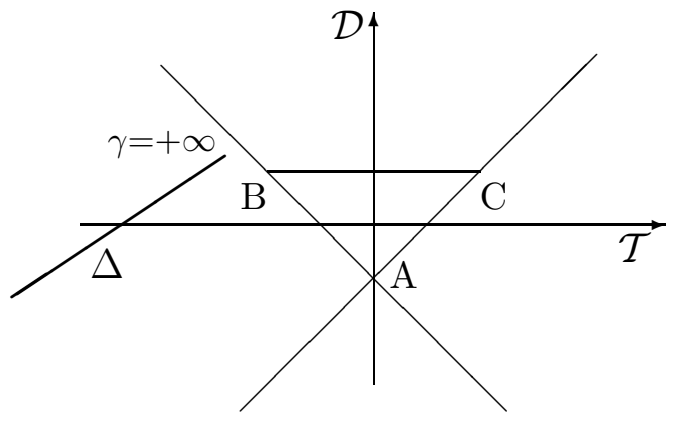

1-ii) $b \in(\bar{b}, 0)$ and $\varepsilon_{r k} \in\left(0, \bar{\varepsilon}_{r k}\right)$

Figure 1: $\Delta$-half-line with $\alpha>\max \{\hat{\alpha}, 1 / 2\}$.

In both cases, saddle-point stability is obtained for any value of the elasticity of intertemporal substitution in consumption $\gamma>1$. However, when $b \in$ $(\bar{b}, 0)$, the elasticity of the rental rate of capital needs to be sufficiently low. 
Consider now the case of intermediary values for $b$. Two different types of configurations may be derived depending on whether $b_{1}$ is lower or larger than $b_{2}$. Let us start in Figure 2 with the case $b_{1}<b_{2}$ which is obtained when $\alpha<s /(1-s)$ and $s>1 / 3$.

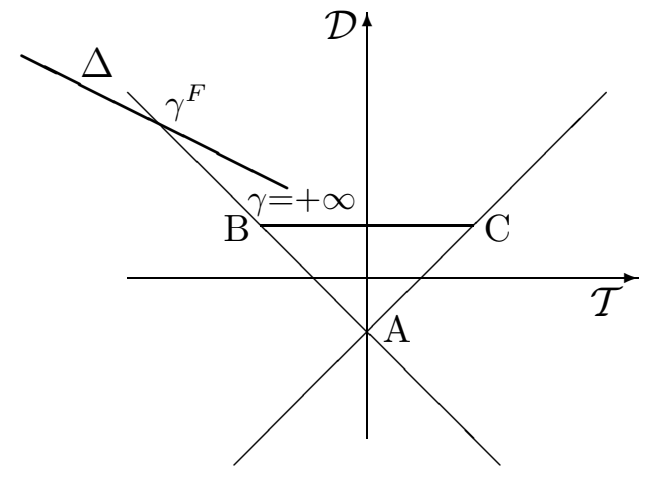

2-i) $b \in\left(-\frac{1}{1+n},-\frac{1-\alpha}{(1+n) \alpha}\right)$

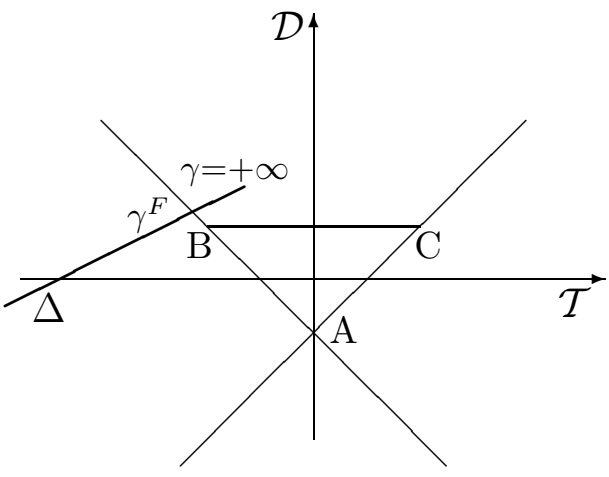

2-ii) $b \in\left(-\frac{1-\alpha}{(1+n) \alpha},-\frac{(1-\alpha)(1-s)}{(1+n) s}\right)$

Figure 2: $\Delta$-half-line with $\alpha \in(\max \{\hat{\alpha}, 1 / 2\}, s /(1-s))$ and $\varepsilon_{r k} \in\left(0, \bar{\varepsilon}_{r k}\right)$.

In both cases, saddle-point stability is obtained for low enough values of the elasticity of the rental rate of capital $\varepsilon_{r k}$ and the elasticity of intertemporal substitution in consumption, $\gamma \in\left(1, \gamma^{F}\right)$ with $\gamma^{F}$ a flip bifurcation value.

Consider finally the case $b_{1}>b_{2}$ which is obtained when $\alpha>s /(1-s)$. If $b \in\left(b_{0}, b_{2}\right)$, we get the same picture as in Figure 2-i) while if $b \in\left(b_{2}, b_{1}\right)$, we get the same picture as in Figure 1-i). It is worth noting at this point that the conditions on $\varepsilon_{r k}$ and $\gamma$ are standard to obtain saddle-point stability. ${ }^{11}$ Moreover, as shown by Figures 1 and 2, they ensure that the characteristic roots cannot equal 1 (since the $\Delta$-half-line cannot cross the line $A C$ ) and thus that the steady state is unique. ${ }^{12}$

\footnotetext{
${ }^{11}$ The same restrictions are also necessary in two-sector optimal growth models to obtain a saddle-point stable steady state (see Benhabib and Nishimura [3], Bosi, Magris and Venditti [5]).

${ }^{12}$ When $\Delta$ crosses the line $A C$, a characterisric root becomes equal to 1 and there generically occurs a transcritical bifurcation implying the existence of two steady states.
} 
Theorem 1. Under Assumptions 1-4, there are two critical bounds $\bar{\varepsilon}_{r k} \in$ $(0,+\infty) \cup\{+\infty\}$ and $\bar{\gamma} \in(1,+\infty) \cup\{+\infty\}$ such that there exists a steady state (the NSS) which is generically unique, dynamic efficiency holds and local indeterminacy is ruled out if $\alpha>\hat{\alpha}, \gamma \in(1, \bar{\gamma})$ and $\varepsilon_{r k} \in\left(0, \bar{\varepsilon}_{r k}\right)$.

Proof: See Appendix 6.6.

As shown in (21), a low value for $\varepsilon_{r k}$ requires large enough sectoral elasticities of capital-labor substitution. Theorem 1 then proves that for a large enough amount of capital-labor substitution, a dynamically efficient steady state cannot be locally indeterminate. ${ }^{13}$

\subsubsection{Dynamic inefficiency and local indeterminacy}

Dynamic inefficiency is obtained when $\alpha \in(0, \hat{\alpha})$. In such a case, $\Delta(\mathcal{T})$ starts within an area in which $\mathcal{D}_{\infty} \in(0,1)$. Different configurations for local indeterminacy may occur depending on whether the starting point $\left(\mathcal{T}_{\infty}, \mathcal{D}_{\infty}\right)$ is within the triangle $A B C$ or not. We are looking for configurations in which local indeterminacy is compatible with reasonable values for the elasticity of the rental rate of capital $\varepsilon_{r k}$. A simple solution consists in considering the cases in which $\left(\mathcal{T}_{\infty}, \mathcal{D}_{\infty}\right)$ belongs to the interior of triangle $A B C$ so that the NSS is locally indeterminate without any restriction on the sectoral elasticities of capital-labor substitution. Assuming that $s \in(0,1 / 3)$, $\alpha \in(s /(1-s), \hat{\alpha})$ and $b \in\left(b_{2}, \min \left\{b_{1}, b_{0}\right\}\right)$, we first get the following geometrical representation in which the $\Delta$-half-line is pointing upward as follows:

\footnotetext{
${ }^{13}$ Considering a parameterization of the model based on US data, we provide in Drugeon, Nourry and Venditti [14] a numerical exercise showing that a dynamically efficient saddle-point stable NSS arises for empirically plausible values for the sectoral elasticities of capital-labor substitution.
} 


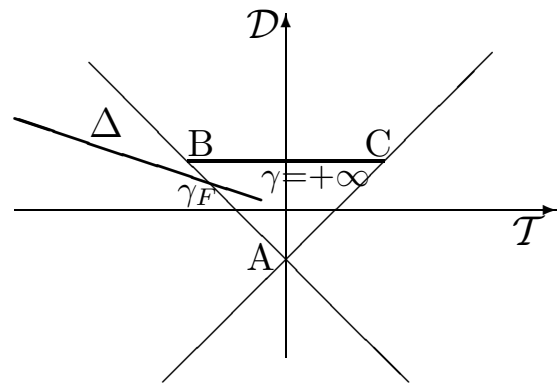

Figure 3: $s \in(0,1 / 3), \alpha \in(s /(1-s), \hat{\alpha})$ and $b \in\left(b_{2}, \min \left\{b_{1}, b_{0}\right\}\right)$.

Local indeterminacy is here based on large enough values for the elasticity of intertemporal substitution in consumption, and uniqueness of the steady state is a generic property since there cannot exist any transcritical bifurcation. However, a flip bifurcation and period-two cycles still occur when $\gamma$ crosses $\gamma^{F}$ from above.

Assume now that $\left(\mathcal{T}_{\infty}, \mathcal{D}_{\infty}\right)$ belongs to the interior of triangle $A B C$, i.e., $b \in\left(b_{2}, b_{0}\right)$, but the $\Delta$-half-line is pointing downward, i.e., $b \in\left(b_{1}, 0\right)$. If $\alpha \in(0, \min \{\hat{\alpha}, 1 / 2\})$ and $b \in\left(\max \left\{b_{1}, b_{2}\right\}, b_{0}\right)$, we get a half-line as given by $\Delta$ or $\Delta^{\prime}$ in the following Figure:

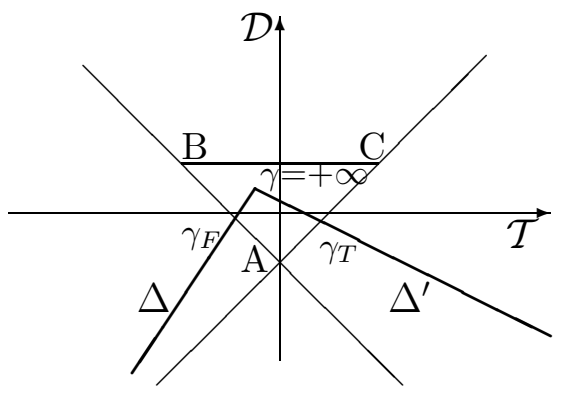

Figure 4: $\alpha \in(0, \min \{\hat{\alpha}, 1 / 2\})$ and $b \in\left(\max \left\{b_{1}, b_{2}\right\}, b_{0}\right)$.

Local indeterminacy is again based on large enough values for the elasticity of intertemporal substitution in consumption. The existence of multiple 
steady states is now a possible outcome since a transcritical bifurcation may occur but it requires low enough values for the sectoral elasticities of capitallabor substitution.

All these results can be gathered into the following Theorem:

Theorem 2. Under Assumptions 1-4, there exist $\underline{\alpha} \in[0, \min \{\hat{\alpha}, 1 / 2\})$, $\bar{\alpha} \in(\underline{\alpha}, \min \{\hat{\alpha}, 1 / 2\}), \underline{b}<\bar{b}<0$ and $\underline{\gamma}>1$ such that the NSS is locally indeterminate if $\alpha \in(\underline{\alpha}, \bar{\alpha}), b \in(\underline{b}, \bar{b})$ and $\gamma>\underline{\gamma}$.

Contrary to the case with dynamic efficiency, under dynamic inefficiency local indeterminacy can arise without any restriction on the sectoral elasticities of capital labor substitution.

Remark 2: There is also a case in which $\left(\mathcal{T}_{\infty}, \mathcal{D}_{\infty}\right)$ belongs to the region where the NSS is saddle-point stable. Indeed, assuming that $\alpha \in$ $(0, \min \{\hat{\alpha}, 1 / 2, s /(1-s)\}), b \in\left(b_{1}, b_{0}\right)$ and $\varepsilon_{r k}>\bar{\varepsilon}_{r k}$, we get the following geometrical representation:

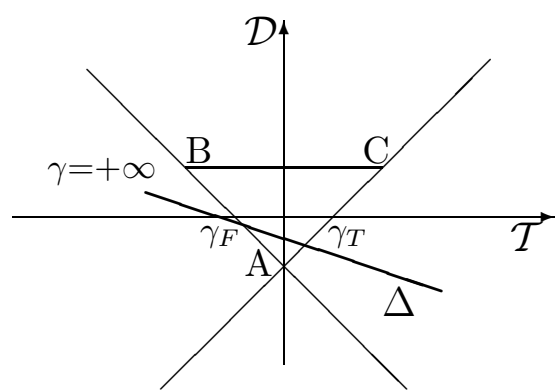

Figure 5: $\alpha \in(0, \min \{\hat{\alpha}, 1 / 2, s /(1-s)\}), b \in\left(b_{1}, b_{0}\right)$ and $\varepsilon_{r k}>\bar{\varepsilon}_{r k}$.

Local indeterminacy requires large enough values for the elasticity of the rental rate of capital, i.e. unplausible low values for the sectoral elasticities of capital-labor substitution, and is associated with the existence of multiple steady states since $\gamma_{T}$ is generically a transcritical bifurcation value. Endogenous fluctuations are also obtained from a flip bifurcation occurring when $\gamma$ crosses $\gamma^{F}$ from below. 


\section{Concluding comments}

We have considered a two-sector two-periods overlapping generations model with inelastic labor, consumption in both periods of life and linearly homogeneous preferences. Under standard conditions on preferences and technologies, we prove that if the share of first period consumption over the wage income is large enough, dynamic efficiency holds and sunspot fluctuations are ruled out. Moreover, as soon as we consider a low enough share of first period consumption over the wage income which implies dynamic inefficiency of the NSS, we show that local indeterminacy arises without requiring strong restrictions on the sectoral elasticities of capital-labor substitution.

The main limitation of our approach results from the local dimension of our dynamic efficiency. Using the Cass [7] criterion, a global analysis of dynamic efficiency could be considered. This is left for future research.

\section{Appendix}

\subsection{Proof of Proposition 1}

Consider the set $\mathcal{K}$ as defined by (12). Then $\kappa^{*} \in(0, \bar{\kappa})$ is a solution of (14)

$$
\alpha\left(-\frac{T_{1}\left(\kappa^{*},(1+n) \kappa^{*}, 1\right)}{T_{2}\left(\kappa^{*},(1+n) \kappa^{*}, 1\right) B}\right)=1+(1+n) \frac{\kappa^{*} T_{2}\left(\kappa^{*},(1+n) \kappa^{*}, 1\right)}{T_{3}\left(\kappa^{*},(1+n) \kappa^{*}, 1\right)} \equiv \Phi_{\kappa^{*}} \in(0,1)
$$

From $(3)$ and $(5)$, we get $R /\left(g^{-1}(R / B) B\right) \equiv \alpha(R / B) /(1-\alpha(R / B))$. Taking elasticities of both sides yields

$$
\alpha^{\prime}(R / B) \frac{R}{\alpha(R / B) B}=(1-\gamma(R / B))(1-\alpha(R / B))
$$

Assumption 2, which is equivalent to $\gamma(R / B)>1$, implies that $\alpha(z)$ is a monotone decreasing function with $\lim _{z \rightarrow 0} \alpha(z)=\alpha_{\text {sup }}, \lim _{z \rightarrow+\infty} \alpha(z)=\alpha_{\text {inf }}$ and $\left(\alpha_{\text {inf }}, \alpha_{\text {sup }}\right) \subseteq(0,1)$. Figure 6 below provides an illustration of $\alpha(z)$. 


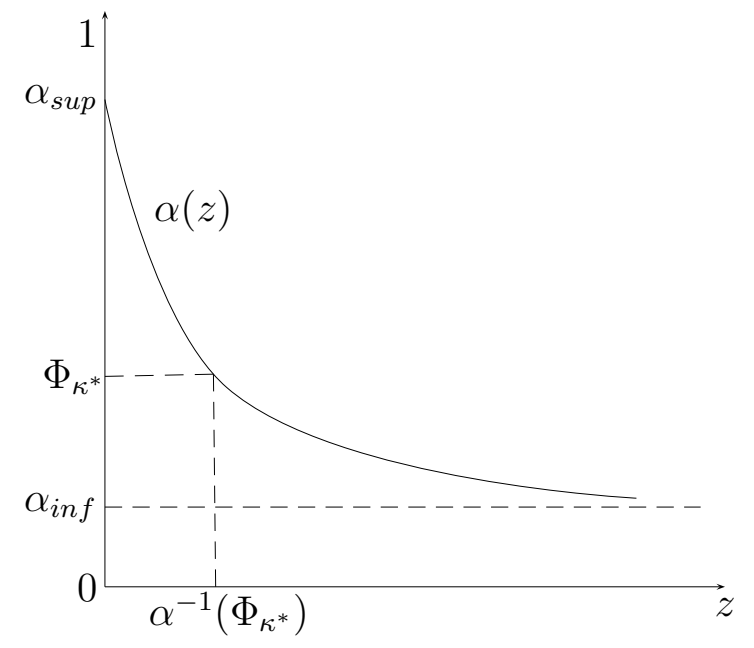

Figure 6: Share of first period consumption $\alpha(z)$.

It follows that $\alpha(z)$ admits an inverse function defined over $\left(\alpha_{\text {inf }}, \alpha_{\text {sup }}\right)$. Let $\kappa^{*} \in(0, \bar{\kappa})$ be such that $\Phi_{\kappa^{*}} \in\left(\alpha_{\text {inf }}, \alpha_{\text {sup }}\right)$. We then derive

$$
B\left(\kappa^{*}\right)=-\frac{T_{1}\left(\kappa^{*},(1+n) \kappa^{*}, 1\right)}{T_{2}\left(\kappa^{*},(1+n) \kappa^{*}, 1\right) \alpha^{-1}\left(\Phi_{\kappa^{*}}\right)}
$$

and $\kappa^{*}$ is a steady state if and only if $B=B\left(\kappa^{*}\right)$.

\subsection{Proof of Proposition 2}

It is shown in Benhabib and Nishimura [3, 4] and Bosi et al. [5] that

$$
T_{12}=-T_{11} b, T_{22}=T_{11} b^{2}<0, T_{31}=-T_{11} a>0, T_{32}=T_{11} a b
$$

with $a \equiv k^{0} / l^{0}>0, b$ as defined by (19) and $T_{11}<0$. Considering that $R(\kappa)=-T_{1}(\kappa,(1+n) \kappa, 1) / T_{2}(\kappa,(1+n) \kappa, 1)$, we derive:

$$
R^{\prime}(\kappa)=-\frac{T_{11}}{T_{2}}[1-(1+n) b](1-R b)
$$

Linear homogeneity of $T(k, y, \ell)$ implies:

$$
a \ell / k=1-(1+n) b>0
$$

Consider the input coefficients in each sector as defined by $a_{00}=l^{0} / y_{0}$, $a_{10}=k^{0} / y_{0}, a_{01}=l^{1} / y$ and $a_{11}=k^{1} / y$. Linear homogeneity of $f^{1}\left(k_{1}, l_{1}\right)$ 
gives $w a_{01}+r a_{11}=p$ or equivalently $p\left(1-R a_{11}\right)=w a_{01}>0$. From the definition of $b$ given by (19) we finally obtain

$$
1-R b=\frac{a_{00}\left(1-R a_{11}\right)+R a_{10} a_{01}}{a_{00}}>0
$$

and $R^{\prime}(\kappa)<0$. Consider now the first order condition for a maximum of the total stationary consumption (18) with respect to $\kappa$ :

$$
-\frac{T_{1}(\kappa,(1+n) \kappa, 1)}{T_{2}(\kappa,(1+n) \kappa, 1)}=1+n
$$

This is equivalent to the equation defining the stationary capital-labor ratio of a two-sector optimal growth model with inelastic labor supply, no discounting and full depreciation of capital. Since $R^{\prime}(\kappa)<0$, the proof of Theorem 3.1 in Becker and Tsyganov [1] applies and there is a unique solution $\hat{\kappa}$ of (30). Along a stationary path of capital stocks, the highest utility is finally defined as the maximum of $u(c, d)$ subject to (18).

\subsection{Proof of Proposition 3}

From (17), we get $R^{*}>1+n$ if and only if $\alpha>\hat{\alpha}$. The rest of the proof is based upon arguments similar to the ones introduced in Chapter 2 (Proposition 2.4, p. 83) of de la Croix and Michel [11]. ${ }^{14}$

Let us start with the case in which the NSS is characterized by an underaccumulation of capital, i.e., $-T_{1}\left(\kappa^{*},(1+n) \kappa^{*}, 1\right) / T_{2}\left(\kappa^{*},(1+n) \kappa^{*}, 1\right)>1+n$. We will show that increasing consumption for one period $t_{1}$ without reducing it at any other period leads to a contradiction. Recall that the ratio of total consumption over labor is given by $\mathcal{C}_{t}=c_{t}+d_{t} /(1+n)=T\left(\kappa_{t},(1+n) \kappa_{t+1}, 1\right)$. Under-accumulation implies that $-T_{1}\left(\kappa^{*},(1+n) \kappa^{*}, 1\right) / T_{2}\left(\kappa^{*},(1+n) \kappa^{*}, 1\right)>$ $z(1+n)$ for some $z>1$. Along a converging equilibrium path we have for

\footnotetext{
${ }^{14}$ We provide an extension to the two-sector framework of the dynamic efficiency property derived within an aggregate Diamond model. The whole argument is based upon the concavity of the social production function $T$ and does not depend on the sign of the capital intensity difference across sectors.
} 
$t$ large enough, say $t \geq t_{0},-T_{1}\left(\kappa_{t},(1+n) \kappa_{t+1}, 1\right) / T_{2}\left(\kappa_{t},(1+n) \kappa_{t+1}, 1\right)>$ $z(1+n)$. At any date $t$, the difference from another feasible path $\tilde{\kappa}_{t}$ satisfies

$$
\Delta \mathcal{C}_{t}=\tilde{\mathcal{C}}_{t}-\mathcal{C}_{t}=T\left(\tilde{\kappa}_{t},(1+n) \tilde{\kappa}_{t+1}, 1\right)-T\left(\kappa_{t},(1+n) \kappa_{t+1}, 1\right)
$$

The concavity of $T$ then implies

$$
\begin{aligned}
\Delta \mathcal{C}_{t} & \leq T_{1}\left(\kappa_{t},(1+n) \kappa_{t+1}, 1\right)\left(\tilde{\kappa}_{t}-\kappa_{t}\right) \\
& +(1+n) T_{2}\left(\kappa_{t},(1+n) \kappa_{t+1}, 1\right)\left(\tilde{\kappa}_{t+1}-\kappa_{t+1}\right) \\
\Leftrightarrow \quad \tilde{\kappa}_{t+1}-\kappa_{t+1} & \leq-\frac{T_{1}\left(\kappa_{t},(1+n) \kappa_{t+1}, 1\right)}{(1+n) T_{2}\left(\kappa_{t},(1+n) \kappa_{t+1}, 1\right)}\left(\tilde{\kappa}_{t}-\kappa_{t}\right)+\frac{\Delta \mathcal{C}_{t}}{(1+n) T_{2}\left(\kappa_{t},(1+n) \kappa_{t+1}, 1\right)}
\end{aligned}
$$

Assume therefore that consumption never decreases. It follows that capital never increases. Indeed, by induction, if $\tilde{\kappa}_{t}-\kappa_{t} \leq 0$, which obviously holds at $t=0$, and if $\Delta \mathcal{C}_{t} \geq 0$ then the previous inequality implies $\tilde{\kappa}_{t+1}-\kappa_{t+1} \leq 0$. Assume moreover that there is some date $t_{1}>0$ such that $\Delta \mathcal{C}_{t_{1}}>0$. Then the previous argument implies $\tilde{\kappa}_{t}-\kappa_{t}<0$ for any $t>t_{1}$ and, for $t>t_{2}=$ $\max \left\{t_{0}, t_{1}\right\}$, we get

$$
\tilde{\kappa}_{t+1}-\kappa_{t+1}<z\left(\tilde{\kappa}_{t}-\kappa_{t}\right)
$$

since $-T_{1}\left(\kappa_{t},(1+n) \kappa_{t+1}, 1\right) / T_{2}\left(\kappa_{t},(1+n) \kappa_{t+1}, 1\right)>z(1+n)$. It follows that

$$
\tilde{\kappa}_{t+1}-\kappa_{t+1}<z^{t-t_{2}}\left(\tilde{\kappa}_{t_{2}}-\kappa_{t_{2}}\right)<0
$$

As $z>1$ and $\kappa_{t+1}$ converges to the NSS, we have the fact that $\tilde{\kappa}_{t+1}-\kappa_{t+1}$ converges to $-\infty$ and $\tilde{\kappa}_{t+1}$ becomes negative, which is not possible.

Consider now the case in which the NSS is characterized by an overaccumulation of capital, i.e., $-T_{1}\left(\kappa^{*},(1+n) \kappa^{*}, 1\right) / T_{2}\left(\kappa^{*},(1+n) \kappa^{*}, 1\right)<1+n$. We will show that we can lower the stock of capital and increase consumption at one date without reducing consumption at another date. Consider an equilibrium path converging to the NSS. In a neighborhood $\left(\kappa^{*}-2 \epsilon, \kappa^{*}+2 \epsilon\right)$ of the NSS we have $-T_{1}(\kappa,(1+n) \kappa, 1) / T_{2}(\kappa,(1+n) \kappa, 1)<1+n$. After some date $t_{0}$, we then have $\kappa_{t} \in\left(\kappa^{*}-\epsilon, \kappa^{*}+\epsilon\right),-T_{1}\left(\kappa_{t},(1+n) \kappa_{t+1}, 1\right) / T_{2}\left(\kappa_{t},(1+\right.$ $\left.n) \kappa_{t+1}, 1\right)<1+n$ and $-T_{1}\left(\kappa_{t}-\epsilon,(1+n)\left(\kappa_{t+1}-\epsilon\right), 1\right) / T_{2}\left(\kappa_{t}-\epsilon,(1+n)\left(\kappa_{t+1}-\right.\right.$ $\epsilon), 1)<1+n$. Let us decrease the stock of capital by $\epsilon$ after date $t_{0}$ and forever. Concavity of $T(k, y, \ell)$ with respect to $y$ implies: 


$$
\begin{aligned}
T\left(\kappa_{t_{0}},(1+n)\left(\kappa_{t_{0}+1}-\epsilon\right), 1\right) & -T\left(\kappa_{t_{0}},(1+n) \kappa_{t_{0}+1}, 1\right) \\
& \geq-T_{2}\left(\kappa_{t_{0}},(1+n)\left(\kappa_{t_{0}+1}-\epsilon\right), 1\right)(1+n) \epsilon
\end{aligned}
$$

Therefore, investment $\kappa_{t_{0}+1}$ is reduced by $\epsilon$ and consumption $T\left(\kappa_{t_{0}},(1+\right.$ $\left.n)\left(\kappa_{t_{0}+1}-\epsilon\right), 1\right)$ is increased by at least $-T_{2}\left(\kappa_{t_{0}},(1+n) \kappa_{t_{0}+1}, 1\right)(1+n) \epsilon$. At $t>t_{0}$ the new consumption is $T\left(\kappa_{t}-\epsilon,(1+n)\left(\kappa_{t+1}-\epsilon\right), 1\right) \equiv \phi(\epsilon)$ with

$$
\begin{aligned}
\phi^{\prime}(\epsilon) & =-T_{1}\left(\kappa_{t}-\epsilon,(1+n)\left(\kappa_{t+1}-\epsilon\right), 1\right) \\
& -(1+n) T_{2}\left(\kappa_{t}-\epsilon,(1+n)\left(\kappa_{t+1}-\epsilon\right), 1\right)
\end{aligned}
$$

Since over-accumulation implies $\phi^{\prime}(\epsilon)>0$ we conclude that consumption can be increased for all periods and the path is dynamically inefficient.

\subsection{Characteristic polynomial}

Lemma 6.1. Under Assumptions 1-3, the characteristic polynomial is

$$
\mathcal{P}(\lambda)=\lambda^{2}-\lambda \mathcal{T}+\mathcal{D}
$$

with

$$
\mathcal{D}=\frac{s[(1+n) b \alpha(\gamma-1)+1-\alpha+\alpha(1+n) b]}{(1+n) b(1-\alpha)(1-s) \alpha(\gamma-1)}, \quad \mathcal{T}=\frac{1}{(1+n) b \varepsilon_{r k} \alpha(\gamma-1)}+\frac{1+\mathcal{D}(1+n)^{2} b^{2}}{(1+n) b}
$$

Proof: Let us define the elasticity of the rental rate of capital

$$
\varepsilon_{r k}=-T_{11}\left(\kappa^{*},(1+n) \kappa^{*}, 1\right) \kappa^{*} / T_{1}\left(\kappa^{*},(1+n) \kappa^{*}, 1\right)
$$

the elasticity of the price of investment good

$$
\varepsilon_{p y}=T_{22}\left(\kappa^{*},(1+n) \kappa^{*}, 1\right)(1+n) \kappa^{*} / T_{2}\left(\kappa^{*},(1+n) \kappa^{*}, 1\right)
$$

and the elasticity of the wage rate

$$
\varepsilon_{w k}=T_{31}\left(\kappa^{*},(1+n) \kappa^{*}, 1\right) \kappa^{*} / T_{3}\left(\kappa^{*},(1+n) \kappa^{*}, 1\right)
$$

all evaluated at the NSS. Total differentiation of (13) using these expressions with (11), (16) and (26) evaluated at the NSS gives

$$
\mathcal{D}=\frac{(1+n) b \varepsilon_{w k}+\varepsilon_{p y}[1+\alpha(\gamma-1)]}{(1+n)^{2} b^{2} \varepsilon_{r k} \alpha(\gamma-1)}, \quad \mathcal{T}=\frac{1+(1+n) b \varepsilon_{w k}+\varepsilon_{p y}+\alpha(\gamma-1)\left(\varepsilon_{r k}+\varepsilon_{p y}\right)}{(1+n) b \varepsilon_{r k} \alpha(\gamma-1)}
$$

Considering (28) with $T_{1} \kappa^{*} / T_{3}=s /(1-s),-T_{1} \kappa^{*} / T_{2}=R^{*}=s /(1-\alpha)(1-s)$ and the fact that $(29)$ implies $a=[1-(1+n) b] \kappa^{*}$ we derive

$$
\varepsilon_{p y}=\frac{\varepsilon_{r k}(1+n)^{2} b^{2} s}{(1-\alpha)(1-s)} \text { and } \varepsilon_{w k}=\frac{\varepsilon_{r k}[1-(1+n) b] s}{(1-s)}
$$

Substituting these expressions into (32) gives the result. 


\subsection{Proof of Proposition 4}

As $\gamma \in(1,+\infty)$, the fundamental properties of $\Delta(\mathcal{T})$ are characterized from the consideration of its extremities. The starting point of the pair $(\mathcal{T}(\gamma), \mathcal{D}(\gamma))$ is indeed obtained when $\gamma=+\infty$ :

$$
\begin{aligned}
\lim _{\gamma \rightarrow+\infty} \mathcal{D}(\gamma) & =\mathcal{D}_{\infty}=\frac{s}{(1-\alpha)(1-s)} \\
\lim _{\gamma \rightarrow+\infty} \mathcal{T}(\gamma) & =\mathcal{T}_{\infty}=\frac{(1-\alpha)(1-s)+(1+n)^{2} b^{2} s}{(1+n) b(1-\alpha)(1-s)}
\end{aligned}
$$

while the end point is obtained when $\gamma=1$ :

$$
\begin{aligned}
& \mathcal{D}(1)=\mathcal{D}_{1}= \pm \infty \Leftrightarrow b[1-\alpha+\alpha(1+n) b] \gtrless 0 \\
& \mathcal{T}(1)=\mathcal{T}_{1}= \pm \infty \\
\Leftrightarrow & b\left[(1-\alpha)(1-s)+\varepsilon_{r k}(1+n) b s[1-\alpha+\alpha(1+n) b]\right] \gtrless 0
\end{aligned}
$$

Moreover, we get

$$
\mathcal{D}^{\prime}(\gamma)=-\frac{s[1-\alpha+\alpha(1+n) b]}{(1+n) b(1-\alpha)(1-s) \alpha(\gamma-1)^{2}}
$$

It follows that $\mathcal{D}^{\prime}(\gamma) \gtrless 0$ if and only if $\mathcal{D}_{1}=\mp \infty$.

The proof of Proposition 4 is organized on the basis of three Lemmas which will be also useful to prove the main Theorems of the paper. The next two Lemmas provide a precise characterization of $\Delta(\mathcal{T})$. The first gives information on the starting point $\left(\mathcal{T}_{\infty}, \mathcal{D}_{\infty}\right)$ and $\mathcal{D}^{\prime}(\gamma)$ :

Lemma 6.2. Under Assumptions 1-4, for given s, $\alpha, b$ and $\varepsilon_{r k}$, the following results hold:

i) $\mathcal{D}_{\infty}>1$ if and only if $\alpha>\hat{\alpha}$;

ii) $\mathcal{D}^{\prime}(\gamma)>0$ if and only if $b \in(-(1-\alpha) /(1+n) \alpha, 0)$;

iii) $\mathcal{T}_{\infty}<0$

iv) When $\alpha>\hat{\alpha}, 1+\mathcal{T}_{\infty}+\mathcal{D}_{\infty}<0$ if and only if $b \in(-\infty,-1 /(1+n)) \cup$ $(-(1-\alpha)(1-s) /(1+n) s, 0)$;

v) When $\alpha \in(0, \hat{\alpha}), 1+\mathcal{T}_{\infty}+\mathcal{D}_{\infty}<0$ if and only if $b \in(-\infty,-(1-$ $\alpha)(1-s) /(1+n) s) \cup(-1 /(1+n), 0)$. 
Proof: i) We get from (17), (33) and Proposition 3 that $\mathcal{D}_{\infty}=R^{*} /(1+$ n) $>1$ iff $\alpha>\hat{\alpha}$.

ii) The result follows from (34) and (35).

iii) The result immediately follows from (33) and Assumption 4. iv)-v) Obvious computations from (33) give

$$
1+\mathcal{T}_{\infty}+\mathcal{D}_{\infty}=[1+(1+n) b] \frac{(1-\alpha)(1-s)+(1+n) b s}{(1+n) b(1-\alpha)(1-s)}
$$

The result follows from the fact that $-(1-\alpha)(1-s) /(1+n) s>-1$ if and only if $\alpha>\hat{\alpha}$.

Lemma 6.2 exhibits three critical bounds on $b$ which appear to be crucial for the stability properties of the NSS: $b_{0}=-1 /(1+n), b_{1}=-(1-\alpha) /(1+n) \alpha$ and $b_{2}=-(1-\alpha)(1-s) /(1+n) s$. We obtain the following comparisons:

$$
\begin{gathered}
b_{1}>b_{0} \Leftrightarrow \alpha>1 / 2, \quad b_{2}>b_{1} \Leftrightarrow \alpha<s /(1-s), \\
\hat{\alpha}<1 / 2 \Leftrightarrow s>1 / 3, \quad s /(1-s)>1 / 2 \Leftrightarrow s>1 / 3
\end{gathered}
$$

A second Lemma then provides additional information on the slope $\mathcal{S}$ and on the intersections of $\Delta(\mathcal{T})$ with the lines $A B$ and $A C$ :

Lemma 6.3. Under Assumptions 1-4, let $\alpha>\hat{\alpha}$ and $b \in\left(b_{1}, 0\right)$. There exists $\bar{\varepsilon}_{r k}>0$ such that for given $s, \alpha, b$ and $\varepsilon_{r k}$, the following results hold:

$1-\Delta(\mathcal{T})=1$ implies $\mathcal{T}<-2$.

2 - $\Delta(\mathcal{T})=-1$ implies $\mathcal{T}<0$ in the following cases:

i) $\alpha>\max \{\hat{\alpha}, 1 / 2\}$,

ii) $s \in(1 / 3,1 / 2), \alpha \in(\hat{\alpha}, 1 / 2)$ and $b \in\left(b_{0}, 0\right)$,

iii) $s \in(1 / 3,1 / 2), \alpha \in(\hat{\alpha}, 1 / 2), b \in\left(b_{1}, b_{0}\right)$ and $\varepsilon_{r k} \in\left(0, \bar{\varepsilon}_{r k}\right)$.

$3-\Delta(\mathcal{T})=-1$ implies $\mathcal{T}>0$ if and only if $s \in(1 / 3,1 / 2), \alpha \in(\hat{\alpha}, 1 / 2)$, $b \in\left(b_{1}, b_{0}\right)$ and $\varepsilon_{r k}>\bar{\varepsilon}_{r k}$.

Proof: Let $b_{0}=-1 /(1+n), b_{1}=-(1-\alpha) /(1+n) \alpha$ and $b_{2}=-(1-$ $\alpha)(1-s) /(1+n) s$.

1 - Solving $\mathcal{D}=1$ in Lemma 6.1 gives 


$$
\alpha(1-\gamma)=\frac{\alpha s\left(b-b_{1}\right)}{b(1-s)(\alpha-\hat{\alpha})}
$$

Under $\alpha>\hat{\alpha}$, since $\gamma>1$, (38) can be satisfied if and only if $b \in\left(b_{1}, 0\right)$. Substituting $\mathcal{D}=1$ into the expression of $\mathcal{T}$ allows to get

$$
\mathcal{T}+2=\frac{1}{(1+n) b \varepsilon_{r k} \alpha(\gamma-1)}+\frac{[1+(1+n) b]^{2}}{(1+n) b}<0
$$

2 - Solving $\mathcal{D}=-1$ in Lemma 6.1 gives

$$
\alpha(1-\gamma)=\frac{\alpha s\left(b-b_{1}\right)}{b[1-\alpha(1-s)]}
$$

Since $\gamma>1$, (40) can be satisfied if and only if $b \in\left(b_{1}, 0\right)$.

i) If $\alpha>1 / 2$ then $b_{1}>b_{0}$ and substituting $\mathcal{D}=-1$ into the expression of $\mathcal{T}$ allows to get under $b \in\left(b_{1}, 0\right)$

$$
\mathcal{T}=\frac{1}{(1+n) b \varepsilon_{r k} \alpha(\gamma-1)}+\frac{1-(1+n)^{2} b^{2}}{(1+n) b}<0
$$

ii) and iii) Now let $s \in(1 / 3,1 / 2)$, so that $\hat{\alpha}<1 / 2$, and $\alpha \in(\hat{\alpha}, 1 / 2)$. It follows that $b_{1}<b_{0}$. Substituting (40) into (41) gives

$$
\mathcal{T}=\frac{\varepsilon_{r k} s\left(b-b_{1}\right)\left[1-(1+n)^{2} b^{2}\right]-b[1-\alpha(1-s)]}{(1+n) b \varepsilon_{r k} \alpha s\left(b-b_{1}\right)}
$$

Therefore when $b \in\left(b_{0}, 0\right), \mathcal{T}<0$ but when $b \in\left(b_{1}, b_{0}\right), \mathcal{T}<0$ if

$$
\varepsilon_{r k}<\frac{b(1-\alpha+\alpha s)}{\left[1-(1+n)^{2} b^{2}\right] \alpha s\left(b-b_{1}\right)} \equiv \bar{\varepsilon}_{r k}
$$

3 - We finally derive from $(42)$ that $\mathcal{D}=-1$ implies $\mathcal{T}>0$ iff $s \in(1 / 3,1 / 2)$, $\alpha \in(\hat{\alpha}, 1 / 2), b \in\left(b_{1}, b_{0}\right)$ and $\varepsilon_{r k}>\bar{\varepsilon}_{r k}$.

Note now from Lemma 6.2 that when $\alpha<\hat{\alpha}$ we have $\mathcal{D}_{\infty} \in(0,1)$ and the existence of local indeterminacy may be obtained either with $\mathcal{D}^{\prime}(\gamma)>0$, i.e. $b \in\left(b_{1}, 0\right)$, or with $\mathcal{D}^{\prime}(\gamma)<0$, i.e. $b \in\left(-\infty, b_{1}\right)$. A third Lemma then provides additional informations on the intersections of $\Delta(\mathcal{T})$ with the lines $A B, B C$ and $A C$ :

Lemma 6.4. Under Assumptions 1-4, let $\alpha \in(0, \hat{\alpha})$. There exists $\bar{\varepsilon}_{r k}>0$ such that for given $s, \alpha, b$ and $\varepsilon_{r k}$, the following results hold:

1 - When $b \in\left(-\infty, b_{1}\right), \Delta(\mathcal{T})=1$ implies $\mathcal{T}<-2$.

2 - When $b \in\left(b_{1}, 0\right), \Delta(\mathcal{T})=-1$ implies $\mathcal{T}<0$ in the following cases: i) $s \in(0,1 / 3)$ and $\alpha \in(1 / 2, \hat{\alpha})$, 
ii) $\alpha \in(0, \min \{\hat{\alpha}, 1 / 2\})$ and $b \in\left(b_{0}, 0\right)$,

iii) $\alpha \in(0, \min \{\hat{\alpha}, 1 / 2\}), b \in\left(b_{1}, b_{0}\right)$ and $\varepsilon_{r k} \in\left(0, \bar{\varepsilon}_{r k}\right)$.

$3-\Delta(\mathcal{T})=-1$ implies $\mathcal{T}>0$ if and only if $\alpha \in(0, \min \{\hat{\alpha}, 1 / 2\})$, $b \in\left(b_{1}, b_{0}\right)$ and $\varepsilon_{r k}>\bar{\varepsilon}_{r k}$.

Proof: Let $\alpha<\hat{\alpha}$. We follow the same steps as in the proof of Lemma 6.3 .

1 - Since $\gamma>1$, we derive from (38) that $\mathcal{D}=1$ can be satisfied if and only if $b \in\left(-\infty, b_{1}\right)$. In such a case we derive from (39) that $\mathcal{D}=1$ implies $\mathcal{T}<-2$. 2 - Since $\gamma>1$, we derive from (40) that $\mathcal{D}=-1$ can be satisfied if and only if $b \in\left(b_{1}, 0\right)$. In such a case we derive from (41) and (42) that $\mathcal{D}=-1$ implies $\mathcal{T}<0$ either when $b>b_{0}$, or when $b<b_{0}$ and $\varepsilon_{r k} \in\left(0, \bar{\varepsilon}_{r k}\right)$, with $\bar{\varepsilon}_{r k}$ as defined by (43). Recall now that $b_{1}>b_{0}$ if and only if $\alpha>1 / 2$, and $\hat{\alpha}>1 / 2$ if and only if $s \in(0,1 / 3)$. We derive from all this that $\mathcal{D}=-1$ implies $\mathcal{T}<0$ in the following cases:

* when $s \in(0,1 / 3), \alpha \in(0,1 / 2)$ and $b \in\left(b_{0}, 0\right)$,

* when $s \in(0,1 / 3), \alpha \in(0,1 / 2), b \in\left(b_{1}, b_{0}\right)$ and $\varepsilon_{r k} \in\left(0, \bar{\varepsilon}_{r k}\right)$,

* when $s \in(0,1 / 3), \alpha \in(1 / 2, \hat{\alpha}), b \in\left(b_{1}, 0\right)$,

* when $s \in(1 / 3,1 / 2), \alpha \in(0, \hat{\alpha}), b \in\left(b_{0}, 0\right)$,

* when $s \in(1 / 3,1 / 2), \alpha \in(0, \hat{\alpha}), b \in\left(b_{1}, b_{0}\right)$ and $\varepsilon_{r k} \in\left(0, \bar{\varepsilon}_{r k}\right)$.

The result follows from summarizing all these subcases.

3 - We finally derive from all the previous subcases that $\mathcal{D}=-1$ implies $\mathcal{T}>0$ iff $\alpha \in(0, \min \{\hat{\alpha}, 1 / 2\}), b \in\left(b_{1}, b_{0}\right)$ and $\varepsilon_{r k}>\bar{\varepsilon}_{r k}$.

Building on all this, we may now prove Proposition 4: If $\alpha>\hat{\alpha}, \Delta(\mathcal{T})$ starts within an area in which local determinacy necessarily holds since $\mathcal{D}_{\infty}>1$. The possible occurrence of local indeterminacy requires therefore that $\mathcal{D}(\gamma)$ is an increasing function. Let us first introduce as suggested by Lemma 6.3 a slightly stronger condition on the share $\alpha$ by assuming that $\alpha>\max \{\hat{\alpha}, 1 / 2\}$. This inequality implies $b_{1}>b_{0}$. While Lemma 6.2 sug- 
gests that local indeterminacy might occur when $b>b_{1}=-(1-\alpha) /(1+n) \alpha$, we derive from Lemma 6.3 that $\mathcal{D}=1$ implies $\mathcal{T}<-2($ see 1$)$ and $\mathcal{D}=-1$ implies $\mathcal{T}<0$ (see 2). As a result, when $\alpha>\max \{\hat{\alpha}, 1 / 2\}, \Delta(\mathcal{T})$ can only cross the line $A B$ when $\mathcal{D}(\gamma)>1$ or the line $A C$ when $\mathcal{D}(\gamma)<-1$ and local indeterminacy is ruled out.

\subsection{Proof of Theorem 1}

The proof of Theorem 1 is organized on the basis of two Propositions. The first one builds upon the determinacy result of Proposition 4 when $\alpha>$ $\max \{\hat{\alpha}, 1 / 2\}$.

Proposition 6.1. Under Assumptions 1-4, let the NSS be characterized by an under-accumulation of capital with $\alpha>\max \{\hat{\alpha}, 1 / 2\}$ and consider the bound $\bar{b}=\max \left\{b_{1}, b_{2}\right\}$. The following results hold:

1 - When $b<b_{0}$, the NSS is generically the unique steady state and is saddle-point stable for any $\gamma>1$;

2- When $b \in\left(b_{0}, \bar{b}\right)$, there is $\bar{\varepsilon}_{r k}>0$ and $\gamma^{F} \in(1,+\infty)$ such that if $\varepsilon_{r k} \in\left(0, \bar{\varepsilon}_{r k}\right)$, the NSS is generically the unique steady state and is saddlepoint stable for $\gamma \in\left(1, \gamma^{F}\right)$, undergoes a flip bifurcation at $\gamma=\gamma^{F}$ and becomes locally unstable for $\gamma>\gamma^{F} .^{15}$ Note that if $b>b_{2}, \gamma^{F}=+\infty$ and the NSS is saddle-point stable for any $\gamma>1$.

3- When $b>\bar{b}$, there is $\bar{\varepsilon}_{r k}>0$ such that if $\varepsilon_{r k} \in\left(0, \bar{\varepsilon}_{r k}\right)$, the NSS is generically the unique steady state and is saddle-point stable for any $\gamma>1$.

Proof: Let $\alpha>\max \{\hat{\alpha}, 1 / 2\}$. From (37), we derive that $b_{1}, b_{2}>b_{0}$. Consider first extreme values for $b$ with $\bar{b}=\max \left\{b_{1}, b_{2}\right\}$ and $b \in\left(-\infty, b_{0}\right) \cup$

\footnotetext{
${ }^{15}$ The period-2 cycles generated by the flip bifurcation are either saddle-point stable if the bifurcation is super-critical, or locally unstable if the bifurcation is sub-critical. As a result, contrary to Cazzavillan and Pintus [8], locally indeterminate periodic cycles cannot occur in this configuration.
} 
$(\bar{b}, 0)$. When $b \in\left(-\infty, b_{0}\right)$, Lemma 6.2 implies $\mathcal{D}^{\prime}(\gamma)<0$ and $1+\mathcal{T}_{\infty}+\mathcal{D}_{\infty}<$ 0 , while Lemma 6.3 implies $\mathcal{S} \in(-1,0)$ for any $\varepsilon_{r k}>0$. We then get Figure $1-i)$ and the uniqueness of the steady state is ensured as no transcritical bifurcation occurs. When $b \in(\bar{b}, 0)$, Lemma 6.2 implies $\mathcal{D}^{\prime}(\gamma)>0$ and $1+\mathcal{T}_{\infty}+\mathcal{D}_{\infty}<0$, while Lemma 6.3 shows that $\mathcal{S} \in(0,1)$ if $\varepsilon_{r k} \in\left(0, \bar{\varepsilon}_{r k}\right)$. Note that this last condition allows to rule out the existence of a transcritical bifurcation. We then get Figure 1-ii).

Consider now the case of intermediary values for $b$. Two different types of configuration may be derived depending on whether $b_{1}$ is lower or larger than $b_{2}$. Let us start in Figure 2 with the case $b_{1}<b_{2}$ which, as shown by (37), is obtained when $\alpha<s /(1-s)$ and $s>1 / 3$. When $b \in\left(b_{0}, b_{1}\right)$, Lemma 6.2 implies $\mathcal{D}^{\prime}(\gamma)<0$ and $1+\mathcal{T}_{\infty}+\mathcal{D}_{\infty}>0$, while Lemma 6.3 shows that $\mathcal{S} \in(0,1)$ if $\varepsilon_{r k} \in\left(0, \bar{\varepsilon}_{r k}\right)$. Note that this last condition is introduced to get the possible existence of saddle-point stability. We then get Figure 2-i) and the uniqueness of the steady state is ensured as no transcritical bifurcation occurs. When $b \in\left(b_{1}, b_{2}\right)$, Lemma 6.2 implies $\mathcal{D}^{\prime}(\gamma)>0$ and $1+\mathcal{T}_{\infty}+\mathcal{D}_{\infty}>0$, while Lemma 6.3 shows that $\mathcal{S} \in(0,1)$ if $\varepsilon_{r k} \in\left(0, \bar{\varepsilon}_{r k}\right)$. Note that this last condition is introduced to rule out the existence of a transcritical bifurcation. We then get Figure 2-ii).

Let us finally consider the case $b_{1}>b_{2}$ which is obtained when $\alpha>$ $s /(1-s)$. If $b \in\left(b_{0}, b_{2}\right)$, we get the same picture as in Figure 2-i): a low elasticity of the rental rate of capital is introduced to get $\mathcal{S} \in(-1,0)$ and thus the existence of saddle-point stability when $\gamma \in\left(1, \gamma^{F}\right)$, with $\gamma^{F}$ a flip bifurcation value. In contrast, if $b \in\left(b_{2}, b_{1}\right)$, we get the same picture as in Figure 1-i): the restriction $\varepsilon_{r k} \in\left(0, \bar{\varepsilon}_{r k}\right)$ implies $\mathcal{S} \in(0,1)$, allows to rule out the existence of a flip bifurcation and ensures saddle-point stability for any $\gamma>1$

We may focus now on the uniqueness property of the steady state. Consider the difference equation (13) as an implicit dynamical system of order 
two parameterized by the elasticity of intertemporal substitution in consumption $\gamma \in(1,+\infty)$ that can be written as $F\left(\kappa_{t}, \kappa_{t+1}, \kappa_{t+2} ; \gamma\right)=0$. Under Assumption 2 and 4 we easily derive that $\partial F / \partial \kappa_{t+2}>0$ and thus there exists a differentiable function $G($.$) which satisfies \kappa_{t+2}=G\left(\kappa_{t}, \kappa_{t+1} ; \gamma\right)$. It follows that the implicit dynamical system can be converted into an explicit dynamical system of order two as

$$
\begin{aligned}
\kappa_{t+1} & =x_{t} \\
x_{t+1} & =G\left(\kappa_{t}, x_{t} ; \gamma\right)
\end{aligned}
$$

Consider then the vector function on the interior of the set $\mathcal{K}$ defined by (12)

$$
H(\kappa, x ; \gamma)=\left(\begin{array}{l}
\kappa-x \\
x-G(\kappa, x ; \gamma)
\end{array}\right)
$$

A steady state of $(44)$ is given by $H(\kappa, x ; \gamma)=0$. Obviously, when $B=B\left(\kappa^{*}\right)$, the NSS satisfies this equation, namely $H\left(\kappa^{*}, \kappa^{*} ; \gamma\right)=0$, for any $\gamma \in(1,+\infty)$. A simple calculation also shows that the characteristic roots solutions of (31) are the eigenvalues of the Jacobian matrix of the right-hand-side of (44) evaluated at the NSS. Thus, if $\lambda_{1}$ and $\lambda_{2}$ are the characteristic roots solutions of $(31)$, the eigenvalues of the Jacobian matrix of $H(\kappa, x ; \gamma)$ evaluated at the NSS will be given by $1-\lambda_{1}$ and $1-\lambda_{2}$. Building on these properties, since $\gamma \in(1,+\infty)$, we can consider $H(\kappa, x ; \gamma)$ as a homotopy on the interior of $\mathcal{K}$ over $(1,+\infty)$ and apply the methodology used by Benhabib and Nishimura [3] (see Theorem 1 and its proof, pp. 288-290) which is based on the property that the topological degree of $H(\kappa, x ; \gamma)$ is a homotopy invariant (see also Guillemin and Pollack [17]). Denoting $Z(\gamma)=\{(\kappa, x) \mid H(\kappa, x ; \gamma)=0\}$, this implies that if the Jacobian matrix of $H(\kappa, x ; \gamma)$ evaluated at the NSS is

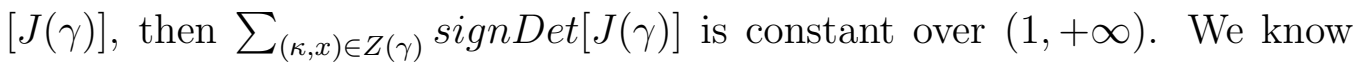
that $\operatorname{Det}[J(\gamma)]=\left(1-\lambda_{1}\right)\left(1-\lambda_{2}\right)$ and from Figures 1 and 2 that when $\varepsilon_{r k} \in\left(0, \bar{\varepsilon}_{r k}\right)$, the characteristic roots $\lambda_{1}$ and $\lambda_{2}$ cannot cross the value 1 as the $\Delta$-half-line never crosses the line $A C$. As a result, the sign of $\operatorname{Det}[J(\gamma)]$ is constant over $(1,+\infty)$ and the NSS is generically the unique steady state. 
This argument relies on the fact that since the transcritical bifurcation is the generic configuration when one characteristic root root crosses 1 , the topological degree evaluated along the NSS would necessarily change if such a bifurcation occurs for some other steady state at some value $\gamma^{T}$. Indeed, the two steady states associated with the transcritical bifurcation are distinct when $\gamma \neq \gamma^{T}$ while they are merged when $\gamma=\gamma^{T}$. It follows that under our restrictions, uniqueness of the steady state is a generic property. ${ }^{16}$

The second Proposition considers the case with $s \in(1 / 3,1 / 2)$ and $\alpha \in$ $(\hat{\alpha}, 1 / 2)$.

Proposition 6.2. Under Assumptions 1-4, let $s \in(1 / 3,1 / 2)$ and the NSS be characterized by an under-accumulation of capital with $\alpha \in(\hat{\alpha}, 1 / 2)$. The following results hold:

1 - When $b<b_{1}$, the NSS is generically the unique steady state and is saddle-point stable for any $\gamma>1$;

2- When $b \in\left(b_{1}, b_{0}\right) \cup\left(b_{2}, 0\right)$, there exists $\bar{\varepsilon}_{r k}>0$ such that if $\varepsilon_{r k} \in\left(0, \bar{\varepsilon}_{r k}\right)$, the NSS is generically the unique steady state and is saddle-point stable for any $\gamma>1$;

3- When $b \in\left(b_{0}, b_{2}\right)$, there exist $\bar{\varepsilon}_{r k}>0$ and $\gamma^{F} \in(1,+\infty)$ such that if $\varepsilon_{r k} \in\left(0, \bar{\varepsilon}_{r k}\right)$, the NSS is generically the unique steady state and is saddlepoint stable for $\gamma \in\left(1, \gamma^{F}\right)$, undergoes a flip bifurcation at $\gamma=\gamma^{F}$ and becomes locally unstable for $\gamma>\gamma^{F}$.

Proof: Let $s \in(1 / 3,1 / 2)$ and $\alpha \in(\hat{\alpha}, 1 / 2)$. We derive from (37) that $b_{1}<b_{0}<b_{2}<0$. Proceeding as in the proof of Proposition 6.1, we easily derive from Lemmas 6.2 and 6.3 that Figure 1-i) illustrates the case $b<b_{1}$,

\footnotetext{
${ }^{16}$ Under Assumptions 2 and 4, it is also possible to show that if $\varepsilon_{r k}$ is low enough, the Jacobian matrix of $H(\kappa, x ; \gamma)$ is non singular over $Z(\gamma)$. As proved in Brock [6] (see Theorem 1, p. 555), since the NSS exists for any $\gamma \in(1,+\infty)$, this property ensures the uniqueness of the steady state.
} 
Figure 1-ii) illustrates the case $b \in\left(b_{1}, b_{0}\right) \cup\left(b_{2}, 0\right)$, and Figure 2-ii) illustrates the case $b \in\left(b_{0}, b_{2}\right)$. In all cases the uniqueness of the steady state is obtained using the same argument as in the proof of Proposition 6.1.

We may now prove Theorem 1. Building on Propositions 6.1 and 6.2, we easily conclude that under Assumptions 1-4, when $\alpha>\hat{\alpha}$, there exist $\bar{\varepsilon}_{r k}>0$ and $\bar{\gamma} \in(1,+\infty)$ such that the NSS is generically the unique steady state and is saddle-point stable for any $\gamma \in(1, \bar{\gamma})$.

\subsection{Proof of Theorem 2}

Proposition 6.3. Under Assumptions 1-4, there exist $\bar{\varepsilon}_{r k}>0, \underline{\gamma}>1$ and $\bar{\gamma}>1$ such that the NSS is locally indeterminate if and only if one of the following sets of conditions is satisfied:

i) $s \in(0,1 / 3), \alpha \in(s /(1-s), \hat{\alpha}), b \in\left(b_{2}, \min \left\{b_{1}, b_{0}\right\}\right)$ and $\gamma>\underline{\gamma}$,

ii) $\alpha \in(0, \min \{\hat{\alpha}, 1 / 2\}), b \in\left(\max \left\{b_{1}, b_{2}\right\}, b_{0}\right)$ and $\gamma>\underline{\gamma}$,

iii) $\alpha \in(0, \min \{\hat{\alpha}, 1 / 2, s /(1-s)\}), b \in\left(b_{1}, b_{0}\right), \varepsilon_{r k}>\bar{\varepsilon}_{r k}$ and $\gamma \in(\underline{\gamma}, \bar{\gamma})$.

Proof: Let us start with the configuration in which $\left(\mathcal{T}_{\infty}, \mathcal{D}_{\infty}\right)$ belongs to the interior of triangle $A B C$ so that the NSS is locally indeterminate. We know from Lemma 6.2 that this is the case when $1+\mathcal{T}_{\infty}+\mathcal{D}_{\infty}>0$, i.e., $b \in\left(b_{2}, b_{0}\right)$.

i) Assume in a first step that the $\Delta$-half-line is pointing upward, i.e., $b \in\left(-\infty, b_{1}\right)$. Lemma 6.4 then shows that $\mathcal{D}=1$ implies $\mathcal{T}<-2$. In order to get a compatibility between $b \in\left(b_{2}, b_{0}\right)$ and $b \in\left(-\infty, b_{1}\right)$, we need to have $b_{1}>b_{2}$, i.e., $\alpha>s /(1-s)$ as shown by (37). Since $\alpha \in(0, \hat{\alpha})$, we have to impose $s /(1-s)<\hat{\alpha}$, or equivalently $s \in(0,1 / 3)$. But this last restriction implies $\hat{\alpha}>1 / 2$ as shown again by (37). Therefore, starting from $\left(\mathcal{T}_{\infty}, \mathcal{D}_{\infty}\right)$ within the interior of triangle $A B C$, and recalling from (37) that $b_{1}>b_{0}$ if 
and only if $\alpha>1 / 2, \Delta(\mathcal{T})$ will cross the segment $A B$ if $s \in(0,1 / 3)$ and one of the following sets of conditions is satisfied:

a) $\alpha \in(s /(1-s), 1 / 2)$ and $b \in\left(b_{2}, b_{1}\right)$,

b) $\alpha \in(1 / 2, \hat{\alpha})$ and $b \in\left(b_{2}, b_{0}\right)$.

To summarize, assuming that $s \in(0,1 / 3), \alpha \in(s /(1-s), \hat{\alpha})$ and $b \in$ $\left(b_{2}, \min \left\{b_{1}, b_{0}\right\}\right)$, we conclude that there exists $\bar{\gamma}>1$ such that the NSS is locally indeterminate if $\gamma>\underline{\gamma}$.

ii) Assume now that $\left(\mathcal{T}_{\infty}, \mathcal{D}_{\infty}\right)$ belongs to the interior of triangle $A B C$, i.e., $b \in\left(b_{2}, b_{0}\right)$, but the $\Delta$-half-line is pointing downward, i.e., $b \in\left(b_{1}, 0\right)$. In order to get a compatibility between $b \in\left(b_{2}, b_{0}\right)$ and $b \in\left(b_{1}, 0\right)$, we need to have $b_{1}<b_{0}$, i.e., $\alpha<1 / 2$ as shown by (37). Let us then assume $\alpha \in(0, \min \{\hat{\alpha}, 1 / 2\})$ and $b \in\left(\max \left\{b_{1}, b_{2}\right\}, b_{0}\right)$. As shown by Lemma 6.4 , depending on whether $\varepsilon_{r k}$ is lower or larger than $\bar{\varepsilon}_{r k}, \mathcal{D}=-1$ implies $\mathcal{T}<0$ or $\mathcal{T}>0$. However, in both cases there exists $\bar{\gamma}>1$ such that the NSS is locally indeterminate if $\gamma>\underline{\gamma}$.

iii) Let us consider finally the configuration in which $\left(\mathcal{T}_{\infty}, \mathcal{D}_{\infty}\right)$ belongs to the region where the NSS is saddle-point stable. We know indeed from Lemma 6.2 that this is the case when $1+\mathcal{T}_{\infty}+\mathcal{D}_{\infty}<0$, i.e., $b \in\left(-\infty, b_{2}\right) \cup$ $\left(b_{0}, 0\right)$. Lemma 6.4 also shows that when $b \in\left(-\infty, b_{1}\right), \mathcal{D}=1$ implies $\mathcal{T}<-2$ so that any $\Delta$-half-line pointing upward cannot intersect the triangle $A B C$. It follows that local indeterminacy requires $\mathcal{D}^{\prime}(\gamma)>0$, i.e., $b \in\left(b_{1}, 0\right)$ as shown by Lemma 6.2. Moreover we derive from Lemma 6.4 that $\Delta(\mathcal{T})$ will cross the triangle $A B C$ if and only if $\alpha \in(0, \min \{\hat{\alpha}, 1 / 2\}), b \in\left(b_{1}, b_{0}\right)$ and $\varepsilon_{r k}>\bar{\varepsilon}_{r k}$, i.e., when $\mathcal{D}=-1$ implies $\mathcal{T}>0$. Note now from (37) that $\alpha \in(0, \min \{\hat{\alpha}, 1 / 2\})$ implies $b_{0}>b_{1}$. Therefore, in order to get a compatibility between $b \in\left(-\infty, b_{2}\right) \cup\left(b_{0}, 0\right)$ and $b \in\left(b_{1}, b_{0}\right)$, we need to have $b_{1}<b_{2}$, i.e., $\alpha<s /(1-s)$ as shown again by (37). To summarize, assuming that $\alpha \in(0, \min \{\hat{\alpha}, 1 / 2, s /(1-s)\}), b \in\left(b_{1}, b_{0}\right)$ and $\varepsilon_{r k}>\bar{\varepsilon}_{r k}$, there exist $\underline{\gamma}>1$ and $\bar{\gamma}>\underline{\gamma}$ such that the NSS is locally indeterminate if $\gamma \in(\underline{\gamma}, \bar{\gamma})$. 
Considering that we focus on the existence of local indeterminacy under plausible conditions on the elasticity of the rental rate of capital $\varepsilon_{r k}$, Theorem 2 obviously follows from cases i) and ii) of Proposition 6.3.

\section{References}

[1] Becker, R. and E. Tsyganov (2002): "Ramsey Equilibrium in a TwoSector Model with Heterogeneous Households," Journal of Economic Theory 105, 188-225.

[2] Benhabib, J. and K. Nishimura (1981): "Stability of Equilibrium in Dynamic Models of Capital Theory," International Economic Review 22, 275-293.

[3] Benhabib, J. and K. Nishimura (1985): "Competitive Equilibrium Cycles," Journal of Economic Theory 35, 284-306.

[4] Benhabib, J. and K. Nishimura (1989): "Stochastic Equilibrium Oscillations," International Economic Review 30, 85-102.

[5] Bosi, S., F. Magris and A. Venditti (2005): "Competitive Equilibrium Cycles with Endogenous Labor," Journal of Mathematical Economics 41, 325-349.

[6] Brock, W. (1973): "Some Results on the Uniqueness of Steady States in Multisector Models of Optimum Growth when Future Utilities are Discounted," International Economic Review 14, 535-559.

[7] Cass, D. (1972): "On Capital Overaccumulation in the Aggregative, Neoclassical Model of Economic Growth: a Complete Characterization," Journal of Economic Theory 4, 200-223. 
[8] Cazzavillan, G. and P. Pintus (2006): "Endogenous Business Cycles and Dynamic Inefficiency," International Journal of Economic Theory 2, 279-294.

[9] Cazzavillan, G. and P. Pintus (2007): "Dynamic Inefficiency in an Overlapping Generations Economy with Production," Journal of Economic Theory 137, 754759 .

[10] Cremers, E. (2006): "Dynamic Efficiency in the Two-Sector Overlapping Generations Model," Journal of Economic Dynamics and Control 30, 1915-1936.

[11] de la Croix, D. and P. Michel (2002): A Theory of Economic Growth: Dynamics and Policy in Overlapping Generations, Cambridge University Press.

[12] Diamond, P. (1965): "National Debt in a Neoclassical Growth Model," American Economic Review 55, 1126-1150.

[13] Drugeon, J.P. (2004): "On Consumptions, Inputs and Outputs Substitutabilities and the Evanescence of Optimal Cycles," Journal of Difference Equations and Applications 10, 473-487.

[14] Drugeon, J.P., C. Nourry and A. Venditti (2006): "Does Dynamic Efficiency Rule Out expectations-driven Fluctuations ?," GREQAM Working Paper.

[15] Galor, O. (1992): "A Two-Sector Overlapping-Generations Model: a Global Characterization of the Dynamical System," Econometrica 60, 1351-1386.

[16] Grandmont, J.M., P. Pintus and R. De Vilder (1998): "Capital-Labor Substitution and Competitive Nonlinear Endogenous Business Cycles," Journal of Economic Theory 80, 14-59. 
[17] Guillemin, V. and A. Pollack (1974): Differential Topology, Prentice Hall.

[18] Kehoe, T. and D. Levine (1985): "Comparative Statics and Perfect Foresight in Infinite Horizon Economies," Econometrica 53, 433-453.

[19] Kehoe, T., D. Levine and P. Romer (1990): "Determinacy of Equilibria in Dynamic Models with Finitely Many Consumers," Journal of Economic Theory 50, 1-21.

[20] Lloyd-Braga, T., C. Nourry and A. Venditti (2007): "Indeterminacy in Dynamic Models: when Diamond Meets Ramsey," Journal of Economic Theory 134, 513-536.

[21] Phelps, E. (1961): "The Golden Rule of Accumulation: A Fable for Growthmen," American Economic Review 51, 638-643.

[22] Reichlin, P. (1992): "Endogenous Fluctuations in a Two-Sector Overlapping Generations Economy," in Benhabib J. (ed.), Cyles and Chaos in Economic Equilibrium, Princeton University Press, Princeton.

[23] Venditti, A. (2005): "The Two Sector Overlapping Generations Model: a Simple Formulation," Research in Economics 59, 164-188.

[24] Woodford, M. (1984): "Indeterminacy of Equilibrium in the Overlapping Generations Model: A Survey," mimeo, Stanford University.

[25] Woodford, M. (1986): "Stationary Sunspot Equilibria: the Case of Small Fluctuations Around a Deterministic Steady State", Mimeo, University of Chicago. 